\title{
Functional Callan-Symanzik Equation for the Coulomb Gas
}

\author{
Sebastiao Correia ${ }^{a *}$, Janos Polonyi ${ }^{a b \dagger}$, Jean Richert ${ }^{a \ddagger}$ \\ ${ }^{a}$ Laboratoire de Physique Théorique ${ }^{\S}$, Université Louis Pasteur \\ 3 rue de l'Université 67084 Strasbourg Cedex, France \\ ${ }^{b}$ Department of Atomic Physics, L. Eötvös University \\ Pázmány P. Sétány 1/A 1117 Budapest, Hungary
}

(November 18, 2018)

\begin{abstract}
A non-perturbative scheme, based on the functional generalization of the Callan-Symanzik equation is developed to treat the Coulomb interaction in an electron gas. The one-particle irreducible vertex functions are shown to satisfy an evolution equation whose initial condition is given by means of the classical action and the final point corresponds to the physical system. This equation is truncated by expanding it in momenta and excitation energies, leaving the electric charge as an arbitrary, not necesseraly small parameter. Exact coupled partial differential equations up to first order in the frequencies and excitation energies are derived. The numerical integration of these eqations is left to a later stage. Nevertheless, in order to demonstrate the relation with the perturbation expansion the one-loop Lindhard function and screening are reproduced in the independent mode approximation of the evolution equation.
\end{abstract}

\section{INTRODUCTION}

In the many electron problem the Coulomb interaction is usually taken into account by a partial resummation of the perturbation expansion. A possible non-perturbative approach, based on the concepts of the renormalization group and the Callan-Symanzik equation is proposed in this paper. Originally viewed as a physically motivated partial resummation of the perturbative contributions [1], the renormalization group strategy has lately developed into a general method to treat strongly coupled systems [2]- [5]. The advantage of this method comes from the functional treatment of the renormalization group flow equation where the only approximation is the truncation of the effective action according to the gradient expansion. This formalism, originating from the Wegner-Houghton equation [2, allows us to follow a large number of coupling constants and makes the approximation rather flexible. Although the Callan-Symanzik [6] equation is not a renormalization group equation because it traces the changes of the dynamics as the mass is varied, its appearance and universality, assumed that the mass is the only scale in the UV regime, show similarity with the renormalization group method.

The traditional application of the renormalization group method for fermions with finite density [7]- [11] represents a promising approach for the treatment of the Coulomb interaction in an electron gas. We present in this paper a version of the functional renormalization group method for systems with Fermi surface which allows for the systematic improvement of the scheme followed in these works. The complication of the blocking in fermionic systems comes from the fact that the successively eliminated modes should be concentrated more and more at the Fermi surface as we follow the renormalization group flow. In the zero density case the infrared limit consists of a single homogeneous mode. But the modes eliminated by the usual blocking in the infrared regime of fermionic systems with finite density populate the Fermi surface. This poses a problem for the implementation of the usual blocking procedure where the interactions are represented by fields. On the one hand, the momentum exchange between electrons is kept finite, of order of $k_{F}$ in the infrared limit, which requires the evolution of these coupling strength. On the other hand, the scalar fields are reduced to the point $p=0$ in the infrared limit and their coupling strength at the scale $k_{F}$ is already frozen out. In other words, the momentum modes of the field variables, responsible of the Coulomb interaction can not be eliminated in a sequential step-by-step manner during the blocking and one can not avoid non-local interactions.

In order to overcome this difficulty we do not order the modes for their elimination according to their energy. Instead, our blocking will eliminate the quantum fluctuations in the increasing order of their amplitude [5]. In this manner we can keep the fundamental requirement of the blocking, namely that it starts with the more weakly coupled

\footnotetext{
*correia@lpt1.u-strasbg.fr

†polonyi@fresnel.u-strasbg.fr

${ }^{\ddagger}$ richert@lpt1.u-strasbg.fr

${ }^{\S}$ Unité Mixte de Recherche CNRS-Université, UMR 7085
} 
modes and treats the more strongly coupled ones later in the renormalization group flow, without paying a high price for the presence of a Fermi surface.

The strategy of this generalized renormalization group method can briefly be summarized as follows. First, one introduces a parameter $M$ in the Hamiltonian in such a way that $H \rightarrow H_{M}$ where $H_{M}$ is almost diagonal in the free particle basis for large $M$, i.e. the interactions are suppressed as $M \rightarrow \infty$. The next step is the computation of the evolution equation in $M$ for the effective action, the generator functional of the one-particle irreducible (1PI) vertices. The renormalization group improved approach consists of a systematic expansion in the vicinity of the current system, namely considering $H_{M}$ as the non-perturbed Hamiltonian and using $\Delta H_{M}=H_{M-\Delta M}-H_{M}$ as perturbation to obtain the changes in the effective action as $M$ is decreased by $\Delta M$. Notice that the exact evolution equation can be obtained already in the leading order of the perturbation expansion as $\Delta M \rightarrow 0$ because $\Delta H_{M}=\mathcal{O}(\Delta M)$, in other words, $\Delta M / M$ is a new small parameter to obtain a differential equation for the $M$-dependence. Once the evolution equation is known, its integration from the perturbative initial condition of the system from large $M$ down to $M=0$ produces the desired 1PI vertices. The evolution equation, being a differential equation for a functional, becomes useful only after projecting it onto a restricted subspace of functionals. In this subspace the effective action is characterized by ordinary functions and the evolution equation is converted into a set of coupled differential equations for them.

This method is based on the following two general assumptions: (i) The dependence of the 1PI functions on the control parameter is supposed to be differentiable. Notice that this condition, the differentiable "turning on" the Coulomb interaction is weaker than the assumption needed for the applicability of the perturbation expansion, the analyticity for arbitrary values of the control parameter. (ii) The possibility of using a simple ansatz for the effective action to incorporate the physics of the model. This step turns the evolution equation, a functional differential equation into a set of coupled differential equations. With these assumptions the perturbation expansion around the Gaussian system is avoided, the 1PI functions are obtained by solving differential equations rather than computing Feynman graphs.

The parameter $M$ controls the amplitude of the quantum fluctuations. In a relativistic system the mass plays such a role and the simplest realization of the blocking in the amplitude is based on the modification $m^{2} \rightarrow m^{2}+M^{2}$ of the Hamiltonian. The resulting differential equation is the functional generalization of the Callan-Symanzik equations [6].

In this paper we present the first step only in the implementation of these ideas in the framework of the Coulomb gas. We consider 1PI functions with the following restrictions: (i) The dependence on the external energies and momenta is kept up to $\mathcal{O}(\omega)$ and $\mathcal{O}(\mathcal{E})$ for electrons where $\omega$ is the frequency and $\mathcal{E}$ is the energy counted from the Fermi surface and up to $\mathcal{O}\left(\omega^{2}\right)$ and $\mathcal{O}\left(p^{2}\right)$ for photons. (ii) There are no more than two external electron legs. The number of photons is not restricted.

We arrive at a set of coupled differential equations in this manner for the functions parametrising the 1PI functions. The numerical integration in the control parameter yields the solution of the Coulomb problem for the assumed ansatz for the 1PI functions. There is no problem in principle to generalize the scheme and include 1PI functions with more external electron lines and more complicated energy and momentum dependence.

In order to demonstrate the relation between the perturbation expansion and our scheme we integrate out the evolution equation in the independent mode approximation. In this approximation the system remains unchanged during the evolution, meaning that the modes are taken into account independently from each other. This corresponds to the one-loop approximation of the perturbation expansion. The one-loop Thomas-Fermi screening and Lindhard function are reproduced in our scheme without considering Feynman graphs.

We derive the evolution equation for the electron gas with Coulomb interaction in section II. The resulting functional differential equation is projected on the leading order gradient expansion ansatz (expansion in $\omega, \mathcal{E}$ and $p$ ) for the effective action in section III. In order to check the consistency of this scheme with the standard perturbation expansion we solve the evolution equations analytically in the independent mode approximation in section IV. Section V contains

the conclusions. Some technical details of the functional derivation and the gradient expansion are shown in the Appendices.

\section{EVOLUTION EQUATION}

We shall study spinless electrons interacting by means of the Coulomb potential and described by the Hamiltonian

$$
H=\int d^{3} x \psi^{\dagger}(x)\left[-\frac{\hbar^{2}}{2 m} \Delta-\mu\right] \psi(x)+\int d^{3} x d^{3} y \psi^{\dagger}(x) \psi^{\dagger}(y) \frac{e^{2}}{4 \pi|x-y|} \psi(y) \psi(x) .
$$


where the field $\psi$ corresponds to electrons and $\mu$ is the chemical potential. In the path integral formalism we introduce an auxiliary field $u(x, t)$, taken formally as the temporal component of the photon field, write the action in terms of the Grassmannian $\psi, \psi^{\dagger}$ and real $u$ as

$$
S\left[\psi, \psi^{\dagger}, u\right]=\int_{x}\left\{\psi_{x}^{\dagger}\left[i \hbar \partial_{t}+\frac{\hbar^{2}}{2 m} \Delta+\mu+e u_{x}\right] \psi_{x}-\frac{1}{2}\left(\nabla u_{x}\right)^{2}\right\}
$$

in the functional integral

$$
\int \mathcal{D}\left[\psi^{\dagger}\right] \mathcal{D}[\psi] \mathcal{D}[u] e^{\frac{i}{\hbar} S\left[\psi, \psi^{\dagger}, u\right]}
$$

The $x, t$ or $p, \omega$ arguments of the field variables will be denoted by the subscripts $x$ and $p$, respectively. The symbols $x$ and $p$ stand for the spatial components only if written otherwise. In a similar manner, we shall use the notation $\int_{x}=\int d t d^{3} x=V T, \int_{p}=\int d \omega d^{3} p /(2 \pi)^{4}$, and $f_{x}=\int_{q} e^{i q \cdot x-i \omega t} f_{q}$. The field $u_{x}$ can be considered either as an auxiliary field, responsible for the Coulomb interaction or the temporal component of the photon field in the Feynman gauge after both the transverse and longitudinal components of the spatial photon field have been suppressed. The homogeneous mode of the photon field,

$$
u_{0}=\frac{1}{V T} \int_{x} u_{x}
$$

plays a special role because of the absence of $\mathcal{O}\left(u_{0}^{2}\right)$ terms in the action. In fact, the homogeneous component of the photon field $u_{0}$ contributes to the chemical potential modifying the electron density and the inhomogeneous field components induce polarization effects only. Another way to see this is to note that the integration over the $u_{0}$ configuration induces the constraint $\int_{x} \psi_{x}^{\dagger} \psi_{x}=0$ and wipes out asymptotic states with non-vanishing electric charge. Actually, the homogeneous photon field configurations in QED, $A_{\mu}(x, t)=a_{\mu}$ are not to be integrated over in the path integral of a non-confining gauge theory [12]. Thus the integral measure $\mathcal{D}[u]$ does not involve the integration over the homogeneous mode i.e. $u_{0}=0$.

We add a quadratic term to the action, $S \rightarrow S_{M}=S+S_{s}$,

$$
S_{s}\left[\psi, \psi^{\dagger}, u\right]=\int_{x}\left\{f\left(M^{2}\right) \psi_{x}^{\dagger}\left(\frac{\hbar^{2}}{2 m} \Delta+\mu\right) \psi_{x}-\frac{M^{2}}{2} u_{x}^{2}\right\},
$$

in order to control the quantum fluctuations. The function $f\left(M^{2}\right)$ is chosen in such a manner that $f(0)=0$ and $\lim _{M \rightarrow \infty} f\left(M^{2}\right)=\infty$, in order to suppress the fluctuations of the fields when $M \rightarrow \infty$. In practice we shall use the simple form $f\left(M^{2}\right)=M^{2} / m^{2}$. Since we are interested in a system of particles at finite density, the chemical potential $\mu$ is non-vanishing. This particular form, (5), was chosen to control the strength of interactions by changing the weight of the quadratic, non-interacting part of the action. The gradual decrease of $S_{s}$ "turns on" the interactions and the integration of the evolution equation in the control parameter allows us to solve the interactive system. The suppression action can be chosen to be gauge invariant, but we are satisfied with this simpler form because the restriction for the Coulomb interaction only, the suppression of the spatial component of the photon field alone removes the gauge symmetry.

The action $S_{M}$ determines the dependence of the microscopic physics on the control parameter $M$. In order to trace down the role played by $M$ we consider the generator functional of the 1PI vertices, the effective potential defined by

$$
\tilde{\Gamma}_{M}\left[\psi, \psi^{\dagger}, u\right]=W_{M}\left[j, j^{\dagger}, J\right]-j^{\dagger} \cdot \psi-\psi^{\dagger} \cdot j-J \cdot u
$$

where $f \cdot g=\int_{x} f_{x} g_{x}$

$$
\tilde{Z}_{M}\left[j, j^{\dagger}, J\right]=e^{\frac{i}{\hbar} W_{M}\left[j, j^{\dagger}, J\right]}=\int \mathcal{D}\left[\psi^{\dagger}\right] \mathcal{D}[\psi] \mathcal{D}[u] e^{\frac{i}{\hbar}\left(S_{M}\left[\psi, \psi^{\dagger}, u\right]+j^{\dagger} \cdot \psi+\psi^{\dagger} \cdot j+J \cdot u\right)},
$$

and

$$
\begin{aligned}
& \frac{\delta W_{M}\left[j, j^{\dagger}, J\right]}{\delta j_{x}}=-\left\langle\psi_{x}^{\dagger}\right\rangle_{j, j^{\dagger}, J}=-\psi_{x}^{\dagger}, \\
& \frac{\delta W_{M}\left[j, j^{\dagger}, J\right]}{\delta j_{x}^{\dagger}}=\left\langle\psi_{x}\right\rangle_{j, j^{\dagger}, J}=\psi_{x}, \\
& \frac{\delta W_{M}\left[j, j^{\dagger}, J\right]}{\delta J_{x}}=\left\langle u_{x}\right\rangle_{j, j^{\dagger}, J}=u_{x} .
\end{aligned}
$$


We use the same notation for the quantum field and its expectation value.

We first derive a functional differential equation in $M^{2}$ for the effective action,

$$
\frac{d \tilde{\Gamma}_{M}}{d\left(M^{2}\right)}=\mathcal{F}\left[\tilde{\Gamma}_{M} ; M\right]
$$

whose integral,

$$
\tilde{\Gamma}_{0}=\tilde{\Gamma}_{M}-\int_{0}^{M^{2}} d\left(M^{\prime}\right)^{2} \mathcal{F}\left[\tilde{\Gamma}_{M^{\prime}} ; M^{\prime}\right]
$$

yields the effective action of the model we want to solve in terms of the initial condition $\tilde{\Gamma}_{M}\left[\psi, \psi^{\dagger}, u\right]=S_{M}\left[\psi, \psi^{\dagger}, u\right]$ imposed when the $M^{2}$ exceeds every energy scale of the model.

The evolution equation of the effective action can be written [5] as

$$
\partial_{M^{2}} \tilde{\Gamma}_{M}\left[\psi, \psi^{\dagger}, u\right]=\partial_{M^{2}} W\left[j, j^{\dagger}, J\right]
$$

where $\partial_{M^{2}}=\partial / \partial M^{2}$ according to $(6)$ and $(8)$. Since we know the control parameter dependence of the bare action we have immediately

$$
\begin{aligned}
\partial_{M^{2}} W_{M} & =-i \hbar e^{-\frac{i}{\hbar} W_{M}} \partial_{M^{2}} e^{\frac{i}{\hbar} W_{M}} \\
& =-f^{\prime}\left(M^{2}\right) \operatorname{Tr}\left\langle\psi_{x}^{\dagger} \mathcal{E} \psi_{x}\right\rangle_{j^{\dagger}, j, J}-\frac{1}{2} \operatorname{Tr}\left\langle u_{x}^{2}\right\rangle_{j^{\dagger}, j, J}
\end{aligned}
$$

where $f^{\prime}=\partial_{M^{2}} f, \mathcal{E}=-\hbar^{2} \Delta / 2 m-\mu$ measures the kinetic energy operator measured from the Fermi level and Tr denotes the functional trace, $\operatorname{Tr} A=\int_{x} A_{x, x}$.

We introduce now the notation

$$
F_{f}^{(1)}=\frac{\delta F[f]}{\delta f}, \quad F_{f g}^{(2)}=\frac{\delta^{2} F[f, g]}{\delta f \delta g}
$$

to relate the second functional derivatives of $W$ and $\tilde{\Gamma}$

$$
\left(\begin{array}{ccc}
\frac{\delta \psi_{z}^{\dagger}}{\delta \psi_{x}^{\dagger}} & \frac{\delta \psi_{z}}{\delta \psi_{x}^{\dagger}} & \frac{\delta u_{z}}{\delta \psi_{x}^{\dagger}} \\
\frac{\delta \psi_{z}^{\dagger}}{\delta \psi_{x}} & \frac{\delta \psi_{z}}{\delta \psi_{x}} & \frac{\delta u_{z}}{\delta \psi_{x}} \\
\frac{\delta \psi_{z}^{\dagger}}{\delta u_{x}} & \frac{\delta \psi_{z}}{\delta u_{x}} & \frac{\delta u_{z}}{\delta u_{x}}
\end{array}\right)=\int_{y}\left(\begin{array}{ccc}
\tilde{\Gamma}_{\psi_{x}^{\dagger} \psi_{y}}^{(2)} & \tilde{\Gamma}_{\psi_{x}^{\dagger} \psi_{y}^{\dagger}}^{(2)} & \tilde{\Gamma}_{\psi_{x}^{\dagger} u_{y}}^{(2)} \\
\tilde{\Gamma}_{\psi_{x} \psi_{y}}^{(2)} & \tilde{\Gamma}_{\psi_{x} \psi_{y}^{\dagger}}^{(2)} & \tilde{\Gamma}_{\psi_{x} u_{y}}^{(2)} \\
\tilde{\Gamma}_{u_{x} \psi_{y}}^{(2)} & \tilde{\Gamma}_{u_{x} \psi_{y}^{\dagger}}^{(2)} & \tilde{\Gamma}_{u_{x} u_{y}}^{(2)}
\end{array}\right)\left(\begin{array}{ccc}
-W_{j_{y} j_{z}}^{(2)} & W_{j_{y} j_{z}^{\dagger}}^{(2)} & W_{j_{y} J_{z}}^{(2)} \\
W_{j_{y} j_{z}}^{(2)} & -W_{j_{y} j_{z}^{\dagger}}^{(2)} & -W_{j_{y} J_{z}}^{(2)} \\
W_{J_{y} j_{z}}^{(2)} & -W_{J_{y} j_{z}^{\dagger}}^{(2)} & -W_{J_{y} J_{z}}^{(2)}
\end{array}\right)=\delta_{x, z} \cdot \mathbf{1}
$$

The evolution equation (11) goes over to

$$
\partial_{M^{2}} \tilde{\Gamma}_{M}\left[\psi^{\dagger}, \psi, u\right]=-i \hbar f^{\prime}\left(M^{2}\right) \operatorname{Tr}\left[\mathcal{E} W_{j, j^{\dagger}}^{(2)}\right]-f^{\prime}\left(M^{2}\right) \operatorname{Tr}\left[\mathcal{E} \psi^{\dagger} \psi\right]+\frac{i \hbar}{2} \operatorname{Tr}\left[W_{J, J}^{(2)}\right]-\frac{1}{2} \operatorname{Tr}\left[u^{2}\right] .
$$

In order to remove the bilinear terms in the fields on the right hand side we write out explicitly the artificial suppression terms in the effective action,

$$
\tilde{\Gamma}_{M}\left[\psi^{\dagger}, \psi, u\right]=\Gamma_{M}\left[\psi^{\dagger}, \psi, u\right]-\int_{x}\left(f\left(M^{2}\right) \psi_{x}^{\dagger} \mathcal{E} \psi_{x}+\frac{1}{2} M^{2} u_{x}^{2}\right)
$$

which leads to

$$
\partial_{M^{2}} \Gamma_{M}\left[\psi^{\dagger}, \psi, u\right]=-i \hbar f^{\prime}\left(M^{2}\right) \operatorname{Tr}\left[\mathcal{E}\left(\Gamma_{M}^{(2)}-\mathcal{M}^{2}\right)_{j, j^{\dagger}}^{-1}\right]+\frac{i \hbar}{2} \operatorname{Tr}\left[\left(\Gamma_{M}^{(2)}-\mathcal{M}^{2}\right)_{J, J}^{-1}\right],
$$

a closed evolution equation for the effective action, with

$$
\mathcal{M}^{2}=\left(\begin{array}{ccc}
-\mathcal{E} & 0 & 0 \\
0 & \mathcal{E} & 0 \\
0 & 0 & M^{2}
\end{array}\right)
$$

Two remarks are in order about details not shown explicitly in Eq. (17): 
- One should bear in mind that the right hand side is UV divergent as it stands because the suppression of the amplitude of the fluctuations is not a regulator. In order to have well defined expressions we introduce a cut-off $\Lambda$ for the momentum integrals. This regulator will be kept finite because our non-relativistic model is non-renormalizable.

- We are working in real time, and the quadratic part of the action must be supplied with an infinitesimal imaginary part. We shall use the electron propagator

$$
G(p, \omega)=\frac{e^{i \omega \eta^{\prime}}}{\omega-\frac{p^{2}}{2 m}+\mu+i \delta_{p}}=\left\{\frac{\Theta\left(k_{F}-p\right)}{\omega-\frac{p^{2}}{2 m}+\mu-i \delta}+\frac{\Theta\left(p-k_{F}\right)}{\omega-\frac{p^{2}}{2 m}+\mu+i \delta}\right\} e^{i \omega \eta^{\prime}},
$$

where $\eta^{\prime} \rightarrow 0^{+}$. Since the chemical potential dependence is not always continuous we have to make sure that $k_{F}$ does not evolve with the control parameter $M^{2}$ in order to avoid the appearance of singularities in the evolution equation. We shall achieve the $M$-independence of the Fermi-momentum by the $\operatorname{choice} \delta_{p}=0^{+} \operatorname{sign}\left(p-k_{F}\right)$. In the case when the chemical potential dependence is continuous then schemes with an $M$-dependent Fermimomentum are possible but their evolution equation appears with an additional term, $\partial \mu / \partial M$ times the density in (12).

\section{GRADIENT EXPANSION}

\section{A. Effective action}

In order to convert the functional differential equation (17) into a set of coupled differential equation for the running coupling constants which are the parameters of the effective action we introduce an ansatz for $\Gamma_{M}\left[\psi^{\dagger}, \psi, u\right]$. We first separate the fluctuations of the photon field from the homogeneous background,

$$
u_{x}=u_{0}+\eta_{x}
$$

where $\eta_{p=0}=0$. Note that once an ansatz is introduced for the effective action we may explore its structure for $u_{0} \neq 0$ even if homogeneous photon field configurations are not allowed in the path integral. Thus $u_{0} \neq 0$ will be used in the effective action whose form will be written as

$$
\begin{aligned}
\Gamma_{M}\left[\psi^{\dagger}, \psi, u\right]= & \int_{x} \psi_{x}^{\dagger}\left[i X_{0}\left(u_{x}\right) \partial_{t}-X_{1}\left(u_{x}\right) \mathcal{E}+U_{1}\left(u_{x}\right)+i \delta_{\mathcal{E}}+\frac{1}{2} Y_{0}\left(u_{x}\right)\left(\partial_{t} u_{x}\right)^{2}+\frac{1}{2} Y_{1}\left(u_{x}\right)\left(\nabla u_{x}\right)^{2}\right] \psi_{x} \\
& +\int_{x}\left[\frac{1}{2} Z_{0}\left(u_{x}\right)\left(\partial_{t} u_{x}\right)^{2}-\frac{1}{2} Z_{1}\left(u_{x}\right)\left(\nabla u_{x}\right)^{2}-U_{2}\left(u_{x}\right)+i \epsilon u_{x}^{2}\right]
\end{aligned}
$$

where $\delta_{\mathcal{E}}=0^{+} \operatorname{sign} \mathcal{E}$ and the $X_{i}, Y_{i}, Z_{i}$ and $U_{i}$ are polynomials in the field $u_{x}$. This funtional form is given in the framework of the gradient expansion which is an expansion in the space-time derivatives of the photon field truncated at $\mathcal{O}\left(\partial^{2}\right)$. We write the effective action in terms of the 1PI functions in momentum space,

$$
\begin{aligned}
\Gamma\left[\psi^{\dagger}, \psi, u_{0}\right]= & \int_{p_{1}, p_{2}} \Gamma_{p_{1}, p_{2}}^{\psi^{\dagger} \psi} \psi_{p_{1}}^{\dagger} \psi_{p_{2}}+\int_{p_{1}, p_{2}, q} \Gamma_{p_{1}, p_{2}, q}^{\psi^{\dagger} \psi u} \psi_{p_{1}}^{\dagger} \psi_{p_{2}} u_{q} \\
& +\frac{1}{2} \int_{q_{1}, q_{2}} \Gamma_{q_{1}, q_{2}}^{u u} u_{q_{1}} u_{q_{2}}+\frac{1}{2} \int_{p_{1}, p_{2}, q_{1}, q_{2}} \Gamma_{p_{1}, p_{2}, q_{1}, q_{2}}^{\psi^{\dagger} \psi u u} \psi_{p_{1}}^{\dagger} \psi_{p_{2}} u_{q_{1}} u_{q_{2}}+\cdots
\end{aligned}
$$

where the superscripts show the type of the external legs and the subscripts give the corresponding momenta. One finds by using this form that the gradient expansion corresponds to the Taylor expansion of the 1PI functions in the photon energy-momentum. In the present work the electronic 1PI functions will be considered by expanding in the frequency and the kinetic energy, $\partial_{t}$ and $\mathcal{E}$, respectively. The dependence on the Grassmannian fermionic field variables is kept quadratic in the present approximation which reduces the argument of the local coefficient functions $X, Y, Z$ and $U$ to the photon field $u$.

The potentials $U_{1}(u)$ and $U_{2}(u)$ are space-time independent, indicating that they do shift the contour of the frequency integrals. The finite lifetime effects might come from the imaginary part of the electron self energy. This appears in $\mathcal{O}\left(\omega^{2}\right)$ and will be ignored in the present work which includes $\mathcal{O}(\omega)$ only.

The infrared divergences of the perturbation expansion generate singular behaviour at $\omega=p=0$. This fact introduces some complications. In particular, the photon 1PI functions are not continuous at this point and their 
limiting value depends on the way this point is approached, as the remnant of gauge invariance, the transversality of the photon self energy. This feature introduces an important difference between the $\mathcal{O}\left(\eta^{0}\right)$ and the $\mathcal{O}\left(\eta^{2}\right)$ level solution of the evolution equations. We have already mentioned after Eq. (4) that the dynamics of the homogeneous mode of the photon field is different from the non-homogeneous ones. To understand better the way this appears in our scheme one must remember that the solution of the complete functional evolution equation for the effective action generates the exact 1PI functions, cf. Eq. (22). As we shall show below, the truncated gradient expansion ansatz for the effective action reduces the evolution equation into a hierarchy of approximate relations between the 1PI functions because only a polynomial of finite order in the external momenta is retained for the 1PI functions in the otherwise exact equation. The integration of the contributions of order $\mathcal{O}\left(\eta^{0}\right)$ to the evolution equation reproduces the value of the 1PI functions at $\omega=p=0$ in this approximation. For example, the integration of the terms $\mathcal{O}\left(\eta^{2}\right)$ yield the photonic two point 1PI function at arbitrary energy-momenta $\Gamma_{u u}^{a p p r}\left(\omega_{1}, p_{1}, \omega_{2}, p_{2}\right)$, but

$$
\lim _{\omega, p \rightarrow 0} \Gamma_{u u}^{a p p r}(\omega, p,-\omega,-p) \neq \Gamma_{u u}^{a p p r}(0,0,0,0) .
$$

We take such a non-continuous energy-momentum dependence of the effective action into account by replacing the potential $U_{2}\left(u_{x}\right)$ in (21) by two different functions,

$$
\int_{x} U_{2}\left(u_{x}\right) \rightarrow \begin{cases}\int_{x} U_{2}\left(u_{0}+\eta_{x}\right) & \eta \neq 0 \\ V T \bar{U}_{2}\left(u_{0}\right) & \eta=0 .\end{cases}
$$

The first line on the right hand side, representing the left hand side in Eq. (23), will be evaluated by taking the limit $\omega \rightarrow 0$ before $p \rightarrow 0$, cf. Eq. (59).

For large enough $M$, when the quantum fluctuations are negligible, we have the initial condition

$$
X_{0}=\hbar, \quad X_{1}=Z_{1}=1, \quad Y_{0}=Y_{1}=U_{2}=\bar{U}_{2}=0, \quad U_{1}=e u,
$$

up to $\mathcal{O}\left(M^{-2}\right)$ corrections because $\Gamma_{M}\left[\psi^{\dagger}, \psi, u\right]=S\left[\psi^{\dagger}, \psi, u\right]$, see after Eq. (10).

\section{B. Expansion in the photon field fluctuations}

In order to determine the evolution equations for the coefficient functions we write the photon field as in Eq. (20) and expand Eq. (17) in powers of $\eta_{x}$. The left hand side is

$$
\Gamma_{M}\left[\psi^{\dagger}, \psi, u\right]=\Gamma_{M}\left[\psi^{\dagger}, \psi, u_{0}\right]+\int_{z} \eta_{z} \Gamma_{M u_{z}}^{(1)}\left[\psi^{\dagger}, \psi, u_{0}\right]+\frac{1}{2} \int_{z, z^{\prime}} \eta_{z} \eta_{z^{\prime}} \Gamma_{M u_{z} u_{z^{\prime}}}^{(2)}\left[\psi^{\dagger}, \psi, u_{0}\right]+\mathcal{O}\left(\eta^{3}\right),
$$

when $\eta \neq 0$. The zeroth order term in $\eta$ gives

$$
\Gamma_{M}\left[\psi^{\dagger}, \psi, u_{0}\right]=\int_{q} \psi_{-q}^{\dagger} \psi_{q} A_{q}-V T \bar{U}_{2},
$$

where $u_{0}$ is an arbitrary parameter, introduced in order to explore our functional differential equation (17),

$$
A_{q}=X_{0} \omega_{q}-X_{1} \mathcal{E}_{q}+U_{1},
$$

the coefficient functions are taken at $u_{0}$, and $\mathcal{E}_{q}=q^{2} / 2 m-\mu$. Note that even though we have $u=u_{0}$ in Eq. (27) we are dealing with the $\eta \neq 0$ part of the effective action because the functional Taylor expansion coefficients are obtained in the limit $\eta \rightarrow 0$. The contributions to the first and second order in $\eta$ can be written as

$$
\int_{q_{1}, q_{2}, q_{3}} \eta_{q_{1}} \psi_{q_{2}}^{\dagger} \psi_{q_{3}} A_{q_{3}}^{(1)} \delta_{q_{1}+q_{2}+q_{3}, 0}
$$

and

$$
\int_{q_{1}, q_{2}, q_{3}, q_{4}} \eta_{q_{1}} \eta_{q_{2}} \psi_{q_{3}}^{\dagger} \psi_{q_{4}}\left[A_{q_{4}}^{(2)}-Y_{0} \omega_{q_{1}} \omega_{q_{2}}-Y_{1} q_{1} \cdot q_{2}\right] \delta_{q_{1}+q_{2}+q_{3}+q_{4}, 0}-\int_{q} \eta_{q} \eta_{-q}\left[Y_{0} \omega_{q}^{2}+Y_{1} q^{2}+U_{2}^{(2)}\right]
$$

by means of the functional derivatives (A1) and (A3) recorded in the Appendix. 
On the right hand side of the evolution equation (17) we find the second functional derivative matrix $\tilde{\Gamma}_{M}^{(2)}=$ $\tilde{\Gamma}_{\mathrm{d}}+\Gamma_{\mathrm{nd}}+\Gamma_{\mathrm{f}}$, where $\tilde{\Gamma}_{\mathrm{d} p_{1}, p_{2}}$ is vanishing for $p_{1}+p_{2} \neq 0, \Gamma_{\mathrm{nd}}=\mathcal{O}\left(\eta^{0}\right)$ and $\Gamma_{\mathrm{fl}}$ comprises the $\mathcal{O}(\eta)$ and $\mathcal{O}\left(\eta^{2}\right)$ contributions. The $\mathcal{O}\left(\eta^{0}\right)$, diagonal piece is obviously

$$
\tilde{\Gamma}_{\mathrm{d}}=\left(\begin{array}{ccc}
-G_{p_{2}}^{-1} & 0 & 0 \\
0 & G_{p_{1}}^{-1} & 0 \\
0 & 0 & D_{p_{1}}^{-1}
\end{array}\right) \delta_{p_{1}+p_{2}, 0},
$$

where

$$
\begin{aligned}
& G_{p}^{-1}=A_{p}-f\left(M^{2}\right) \mathcal{E}_{p}+i \delta_{\mathcal{E}_{p}}, \\
& D_{p}^{-1}=Z_{0} \omega_{p}^{2}-Z_{1} p^{2}-U_{2}^{(2)}-M^{2}+i \epsilon .
\end{aligned}
$$

Note that the $U_{1}$ of Eq. (28) does not influence the imaginary part of $G$ and the Fermi level remains independent of the photon field. This is in agreement with our introduction of $u_{0}$ in the effective action as a formal parameter to help to uncover the content of the functional equation (12) rather than a dynamical variable.

The remaining $\mathcal{O}\left(\eta^{0}\right)$ contributions are easily found,

$$
\Gamma_{\text {nd }}=\left(\begin{array}{ccc}
0 & 0 & \Sigma^{\psi^{\dagger} u} \\
0 & 0 & \Sigma^{\psi u} \\
\Sigma^{u \psi} & \Sigma^{u \psi^{\dagger}} & \Sigma^{u u}
\end{array}\right)
$$

the matrix elements being listed in Eq. A5). The terms $\mathcal{O}(\eta)$ and $\mathcal{O}\left(\eta^{2}\right)$, parametrized as

$$
\Gamma_{\mathrm{fl}}=\left(\begin{array}{ccc}
\sigma^{\psi^{\dagger} \psi} & \sigma^{\psi^{\dagger} \psi^{\dagger}} & \sigma^{\psi^{\dagger} u} \\
\sigma^{\psi \psi} & \sigma^{\psi \psi^{\dagger}} & \sigma^{\psi u} \\
\sigma^{u \psi} & \sigma^{u \psi^{\dagger}} & \sigma^{u u}
\end{array}\right)+\mathcal{O}\left(\eta^{3}\right),
$$

are explicitly presented in Eq. A9.

The inversion of $\Gamma^{(2)}$ is carried out by expanding in the non-diagonal pieces,

$$
W^{(2)}=W_{0}^{(2)}+\sum_{\alpha_{1}} W_{\alpha_{1}}^{(2)}+\sum_{\alpha_{1}, \alpha_{2}} W_{\alpha_{1}, \alpha_{2}}^{(2)}+\sum_{\alpha_{1}, \alpha_{2}, \alpha_{3}} W_{\alpha_{1}, \alpha_{2}, \alpha_{3}}^{(2)}+\sum_{\alpha_{1}, \alpha_{2}, \alpha_{3}, \alpha_{4}} W_{\alpha_{1}, \alpha_{2}, \alpha_{3}, \alpha_{4}}^{(2)}+\cdots
$$

where the summation is over the indices $\alpha_{j}=$ nd or fl and $W_{0}^{(2)}=\Gamma_{\mathrm{d}}^{-1}$ denotes the diagonal part of the propagator. The non-diagonal contributions are collected in

$$
\begin{aligned}
W_{\alpha_{1}}^{(2)} & =-W_{0}^{(2)} \Gamma_{\alpha_{1}} W_{0}^{(2)} \\
W_{\alpha_{1}, \alpha_{2}}^{(2)} & =W_{0}^{(2)} \Gamma_{\alpha_{1}} W_{0}^{(2)} \Gamma_{\alpha_{2}} W_{0}^{(2)}, \\
W_{\alpha_{1}, \alpha_{2}, \alpha_{3}}^{(2)} & =W_{0}^{(2)} \Gamma_{\alpha_{1}} W_{0}^{(2)} \Gamma_{\alpha_{2}} W_{0}^{(2)} \Gamma_{\alpha_{3}} W_{0}^{(2)} \\
W_{\alpha_{1}, \alpha_{2}, \alpha_{3}, \alpha_{4}}^{(2)} & =-W_{0}^{(2)} \Gamma_{\alpha_{1}} W_{0}^{(2)} \Gamma_{\alpha_{2}} W_{0}^{(2)} \Gamma_{\alpha_{3}} W_{0}^{(2)} \Gamma_{\alpha_{4}} W_{0}^{(2)} .
\end{aligned}
$$

In the last step we substitute the gradient expanded form, (21), and identify in (17) the contributions of the different powers in the electron field and the fluctuations in order to get the evolution equations for the coefficient functions.

\section{Homogeneous photon field}

The zeroth order in the fluctuations is obtained by setting $\eta=0$ on both sides of (17). On the right hand side, in particular in Eq. (35) this amounts to neglect terms containing $\Gamma_{\mathrm{ff}}$. The rest involves the expressions

$$
\operatorname{Tr}\left[\mathcal{E}\left(\Gamma_{M}^{(2)}-\mathcal{M}^{2}\right)_{j, j^{\dagger}}^{-1}\right]=-\operatorname{Tr}\left[\mathcal{E} W_{j, j^{\dagger}}^{(2)}\right]=\int_{p} \mathcal{E}_{p} G_{p}-\int_{p} \int_{p^{\prime}} \mathcal{E}_{p} G_{p}^{2} \Sigma_{-p, p^{\prime}}^{\psi, u} D_{p^{\prime}} \Sigma_{-p^{\prime}, p}^{u, \psi^{\dagger}},
$$

and 


$$
\begin{aligned}
\operatorname{Tr}\left[\left(\Gamma_{M}^{(2)}-\mathcal{M}^{2}\right)_{J, J}^{-1}\right] & =\operatorname{Tr}\left[W_{J, J}^{(2)}\right] \\
& =\int_{p}\left[D_{p}-D_{p}^{2} \Sigma_{-p, p}^{u, u}\right]+\int_{p, p^{\prime}} D_{p}^{2}\left[\Sigma_{-p, p^{\prime}}^{u, \psi} G_{p^{\prime}} \Sigma_{-p^{\prime}, p}^{\psi^{\dagger}, u}-\Sigma_{-p, p^{\prime}}^{u, \psi^{\dagger}} G_{p^{\prime}} \Sigma_{-p^{\prime}, p}^{\psi, u}-\Sigma_{-p, p^{\prime}}^{u, u} D_{p^{\prime}} \Sigma_{-p^{\prime}, p}^{u, u}\right]
\end{aligned}
$$

The left hand side of the evolution equation (17) includes the expression (27) and the use of (32) and (A5) gives the evolution equations

$$
\begin{aligned}
& \partial_{M^{2}} \bar{U}_{2}=i \hbar f^{\prime} \int_{p} \frac{\mathcal{E}_{p}}{X_{0} \omega_{p}-\left(X_{1}+f\right) \mathcal{E}_{p}+U_{1}}-i \hbar \int_{p} \frac{1}{Z_{0} \omega_{p}^{2}-Z_{1} p^{2}-U_{2}^{(2)}-M^{2}}, \\
& \partial_{M^{2}}\left[X_{0} \omega_{q}-X_{1} \mathcal{E}_{q}+U_{1}\right] \underline{\underline{\underline{g}}}-\frac{i \hbar}{2} \int_{p} \frac{A_{q}^{(2)}+Y_{0} \omega_{p}^{2}+Y_{1} p^{2}}{\left(Z_{0} \omega_{p}^{2}-Z_{1} p^{2}-U_{2}^{(2)}-M^{2}\right)^{2}} \\
& +\frac{i \hbar}{2} A_{q}^{(1)} \int_{p} \frac{A_{-p}^{(1)}}{\left(Z_{0} \omega_{p+q}^{2}-Z_{1}(p+q)^{2}-U_{2}^{(2)}-M^{2}\right)^{2}\left(X_{0} \omega_{p}-\left(X_{1}+f\right) \mathcal{E}_{p}+U_{1}\right)} \\
& +i \hbar f^{\prime} A_{q}^{(1)} \int_{p} \frac{\mathcal{E}_{p} A_{-p}^{(1)}}{\left(X_{0} \omega_{p}-\left(X_{1}+f\right) \mathcal{E}_{p}+U_{1}\right)^{2}\left(Z_{0} \omega_{p+q}^{2}-Z_{1}(p+q)^{2}-U_{2}^{(2)}-M^{2}\right)} .
\end{aligned}
$$

We introduced the symbol $\underline{g}$ to indicate that the right hand side is supposed to be written by expanding in the variables $\omega, \mathcal{E}$ and $p$ and by retaining only the orders shown on the left hand side.

As we shall see below, the discontinuity discussed in Eq. (23) affects only Eq. (39). Eq. (40) gives the evolution of the coefficents $X_{0}, X_{1}$ and $U_{1}$. In order to solve these coupled differential equations one needs other equations for the $Z_{i}$ 's, $Y_{i}$ 's and $U_{2}$.

\section{Fluctuations}

In order to find the evolution of the coefficient functions we substitute the gradient expanded form, (21), and identify on the right hand side of Eq. (17) the contributions of different powers in $\psi^{\dagger} \psi$ and $\eta$. Some expressions of the contributions of the individual terms on the right hand side of Eq. (35) are shown in Appendix B.

It is easy to isolate the terms $\mathcal{O}\left(\left(\psi^{\dagger} \psi\right)^{0} \eta^{2}\right)$,

$$
\begin{aligned}
& \frac{1}{2}\left(\partial_{M^{2}} Z_{0} \omega_{q}^{2}-\partial_{M^{2}} Z_{1} q^{2}-\partial_{M^{2}} U_{2}^{(2)}\right) \underline{\mathrm{gr}} \\
& \quad i \hbar f^{\prime} \int_{p} \frac{\mathcal{E}_{p} A_{p}^{(1)} A_{p+q}^{(1)}}{\left(X_{0} \omega_{p}-\left(X_{1}+f\right) \mathcal{E}_{p}+U_{1}\right)^{2}\left(X_{0} \omega_{p+q}-\left(X_{1}+f\right) \mathcal{E}_{p+q}+U_{1}\right)} \\
& \quad+i \frac{\hbar f^{\prime}}{2} \int_{p} \frac{\mathcal{E}_{p}\left(A_{p}^{(2)}-Y_{0} \omega_{q}^{2}+Y_{1} q^{2}\right)}{\left(X_{0} \omega_{p}-\left(X_{1}+f\right) \mathcal{E}_{p}+U_{1}\right)^{2}}-\frac{i \hbar}{4} \int_{p} \frac{Z_{0}^{(2)}\left(\omega_{q}^{2}+\omega_{p}^{2}\right)+Z_{1}^{(2)}\left(q^{2}+p^{2}\right)+U_{2}^{(4)}}{\left(Z_{0} \omega_{p}^{2}-Z_{1} p^{2}-U_{2}^{(2)}-M^{2}\right)^{2}} \\
& \quad-\frac{i \hbar}{2} \int_{p} \frac{\left[Z_{0}^{(1)}\left(\omega_{p}^{2}+\omega_{q}^{2}+\omega_{p} \omega_{q}+Z_{1}^{(1)}\left(p^{2}+q^{2}+p \cdot q\right)+U_{2}^{(3)}\right]^{2}\right.}{\left(Z_{0} \omega_{p}^{2}-Z_{1} p^{2}-U_{2}^{(2)}-M^{2}\right)^{2}\left(Z_{0} \omega_{p+q}^{2}-Z_{1}(p+q)^{2}-U_{2}^{(2)}-M^{2}\right)} .
\end{aligned}
$$

The discontinuity (23) appears here as the non-commutivity of the integration and the limit $\omega_{q}, q \rightarrow 0$ in this equation. The singularity at $q=0$ is caused by the fact that the order of the singularity in $\omega_{p}$ is 2 or 3 when $q=0$ or $q \neq 0$. Since we discuss the $\mathcal{O}\left(\eta^{2}\right)$ contributions we have to keep $q \neq 0$. The $q$-independent part of the right hand side, the extrapolation from $q \neq 0$ to $q=0$ will contribute to $U_{2}^{(2)}$ rather than $\bar{U}_{2}^{(2)}$ according to Eq.(24).

The terms $\mathcal{O}\left(\psi^{\dagger} \psi \eta\right)$ on the left hand side of Eq. (17) is

$$
\partial_{M^{2}} \int_{q_{1}, q_{2}} \eta_{q_{1}} \psi_{-q_{1}-q_{2}}^{\dagger} \psi_{q_{2}}\left[X_{0}^{(1)} \omega_{q_{2}}-X_{1}^{(1)} \mathcal{E}_{q_{2}}+U_{1}^{(1)}\right]
$$


Since we have $q_{1} \neq 0$ there will be no singular coincidence of the poles on the right hand side of the evolution equation. As a result the terms $\mathcal{O}\left(\omega_{q_{2}}^{0} \mathcal{E}_{q_{2}}^{0}\right), \mathcal{O}\left(\omega_{q_{2}} \mathcal{E}_{q_{2}}^{0}\right)$ and $\mathcal{O}\left(\omega_{q_{2}}^{0} \mathcal{E}_{q_{2}}\right)$ on the right hand side of the evolution equation of order $\mathcal{O}\left(\psi^{\dagger} \psi \eta\right)$ reproduce the first derivative of the right hand side of Eq. (40) with respect to $u_{0}$.

In the order $\mathcal{O}\left(\psi^{\dagger} \psi \eta^{2}\right)$ we find

$$
\frac{1}{2} \partial_{M^{2}} \int_{q_{1}, q_{2}, q_{3}} \eta_{q_{1}} \eta_{q_{2}} \psi_{-q_{1}-q_{2}-q_{3}}^{\dagger} \psi_{q_{3}}\left[X_{0}^{(2)} \omega_{q_{3}}-X_{1}^{(2)} \mathcal{E}_{q_{3}}+U_{1}^{(2)}-Y_{0} \omega_{q_{1}} \omega_{q_{2}}-Y_{1} q_{1} \cdot q_{2}\right]
$$

on the left hand side of the evolution equation. The terms $\mathcal{O}\left(\omega_{q_{3}}\right), \mathcal{O}\left(\mathcal{E}_{q_{3}}\right)$ and $\mathcal{O}(1)$ give the second derivatives of Eq. (40) and can be omitted on both sides of the equation. The remaining contribution in $\mathcal{O}\left(\omega_{q_{1}} \omega_{q_{2}}\right)$ and $\mathcal{O}\left(q_{1} \cdot q_{2}\right)$, obtained for $q_{3}=0$ is

$$
\begin{aligned}
& \frac{1}{2} \partial_{M^{2}} \int_{q_{1}, q_{2}, q_{3}} \eta_{q_{1}} \eta_{q_{2}} \psi_{-q_{1}-q_{2}-q_{3}}^{\dagger} \psi_{q_{3}}\left[Y_{0} \omega_{q_{1}} \omega_{q_{2}}+Y_{1} q_{1} \cdot q_{2}\right] \\
& \stackrel{\underline{\underline{\operatorname{gr}}}}{i} i \hbar\left(\operatorname{Tr}\left[\mathcal{E} W_{\mathrm{fl}, j, j^{\dagger}}\right]-\mathcal{F}_{2}-\mathcal{F}_{3}-\mathcal{F}_{4}\right)-f\left(M^{2}\right)\left(\operatorname{Tr}\left[W_{\mathrm{fl}, J, J}\right]+\mathcal{B}_{2}+\mathcal{B}_{3}+\mathcal{B}_{4}\right),
\end{aligned}
$$

where the quantities on the right hand side are given in Appendix B. When the coefficients of the field variables with the same arguments are identified on both side we find two differential equations, one for $Y_{0}$ and another for $Y_{1}$.

The form of these and the other differential equations for the coefficient functions can simply be understood in the following manner. We start with the one-loop expressions for the 1PI vertex functions using the non-Gaussian parts of the effective action $\tilde{\Gamma}$ as interaction. The infinitesimal increase of the parameter $M^{2} \rightarrow M^{2}+\delta M^{2}$ modifies the electron and photon propagators as

$$
G \rightarrow G-\delta M^{2} f^{\prime}\left(M^{2}\right) G^{2}, \quad D \rightarrow D-\delta M^{2} D^{2},
$$

respectively. The change induced by this transformation in the one-loop expressions for the 1PI vertex functions is the right hand side of our evolution equation up to the factor $\delta M^{2}$. At this stage we recover the perturbation expansion based on the Feynman rules. The usual accumulation effect of the renormalization group strategy occurs when the differential equations are integrated out. By making $n$ steps $M^{2} \rightarrow M^{2}+\delta M^{2}$ the integration sums up the $n$-loop contributions to the corresponding 1PI vertex function.

In this manner the integration of the evolution equations Eqs. (39), (40), (41) and (44) with the initial condition (25) provides the effective action, the 1PI functions in the given approximation of their dependence on the external momenta. The successive inclusion of terms $\mathcal{O}\left(\left(\psi^{\dagger} \psi\right)^{n}\right)$ or higher order derivatives in the ansatz (21) for the effective action yields more complete solutions.

\section{INDEPENDENT MODE APPROXIMATION}

The renormalization group strategy is based on the accumulation of the effects of the modes which are eliminated sequentially. This corresponds to the integration of the evolution equations where the right hand side evolves with $M^{2}$. We can easily obtain an analytic approximation for the evolution by ignoring the $M^{2}$-dependence on the right hand side of the evolution equation. This amounts to the independent mode approximation which reproduces the one-loop 1PI functions in the given order of the external momenta.

\section{A. Homogeneous photon field: Hartree-Fock approximation}

As already mentioned in paragraph III A, the 1PI functions are non-analytic at $\omega=p=0$. We shall first show that this implies that $\bar{U}_{2}$ does not evolve. The right hand side of Eq. (17) is obtained by using an $M^{2}$-independent effective action (21), given by Eq. (25). As far as the $\eta=0$ case is concerned we find

$$
\partial_{M^{2}} \bar{U}_{2}=-i \hbar f^{\prime} \int_{p} \frac{\mathcal{E}_{p}}{\hbar \omega_{p}-(1+f) \mathcal{E}_{p}+e u_{0}}-i \hbar \int_{p} \frac{1}{p^{2}+M^{2}}
$$

After the frequency integration of the first term,

$$
\lim _{\eta \rightarrow 0} \int \frac{d^{3} p}{(2 \pi)^{3}} \mathcal{E}_{p} \int \frac{d \omega}{2 \pi} \frac{e^{i \omega \eta}}{\hbar \omega_{p}-(1+f) \mathcal{E}_{p}+e u_{0}+i \delta_{p}}=\int \frac{d^{3} p}{(2 \pi)^{3}} \mathcal{E}_{p} i \Theta\left(-\mathcal{E}_{p}\right)=-i \frac{k_{F}^{5}}{30 \pi^{2} m},
$$


the right hand side turns out to be $u_{0}$-independent. Since we are interested in the field dependent part of the effective action only, Eq. (46) gives $\partial_{M^{2}} \bar{U}_{2}=0$. Polarization effects do not appear for a homogeneous field.

The non-analyticity of the effective action at $\omega=p=0$ is due to the photon sector only. The electron sector can be studied in the homogeneous photon field approximation, $\eta=0$ in Eq. (20). The identification of the contributions $\mathcal{O}\left(\psi^{\dagger} \psi\right)$ in Eq. (17), the use of Eqs (28) and (40) taken at the one-loop approximation and the initial condition Eq. (25) lead to

$$
\begin{array}{r}
\partial_{M^{2}}\left[X_{0} \omega_{q}-X_{1} \mathcal{E}_{q}+U_{1}\right] \stackrel{\text { gr }}{\stackrel{i}{i}} \\
+i \hbar f^{\prime} e^{2} \int_{p} \frac{\mathcal{E}_{p}}{\left(\hbar \omega_{p}-(1+f) \mathcal{E}_{p}+e u_{0}\right)^{2}\left((p+q)^{2}+M^{2}\right)} \frac{1}{\left(\hbar \omega_{p}-(1+f) \mathcal{E}_{p}+e u_{0}\right)\left((p+q)^{2}+M^{2}\right)^{2}}
\end{array}
$$

The frequency integral in the first term appearing in Eq. (48) vanishes. The second term gives

$$
-e^{2} \int \frac{d^{3} p}{(2 \pi)^{3}} \frac{\Theta\left(-\mathcal{E}_{p}\right)}{\left((p+q)^{2}+M^{2}\right)^{2}}=-\frac{e^{2}}{2 \pi^{2}} \int_{0}^{k_{F}} \frac{p^{2} d p}{\left(p^{2}+q^{2}+M^{2}\right)^{2}-4 p^{2} q^{2}} .
$$

In order to identify the $\mathcal{O}\left(\omega_{q}\right), \mathcal{O}\left(\mathcal{E}_{q}\right)$ and $\mathcal{O}(1)$ terms with those appearing in the left hand side of Eq. (48), the last expression is expanded in powers of $\mathcal{E}_{q}$,

$$
-\frac{e^{2}}{2 \pi^{2}} \int_{0}^{k_{F}} \frac{p^{2} d p}{\left(p^{2}+k_{F}^{2}+M^{2}\right)^{2}-4 p^{2} k_{F}^{2}}\left(1-\frac{4 m\left(-p^{2}+k_{F}^{2}+M^{2}\right)}{\left(p^{2}+k_{F}^{2}+M^{2}\right)^{2}-4 p^{2} k_{F}^{2}} \mathcal{E}_{q}+\mathcal{O}\left(\mathcal{E}_{q}^{2}\right)\right) .
$$

The identification of the different terms in Eq. (48) leads to the evolution equations $\partial_{M^{2}} X_{0}=0$, and

$$
\begin{aligned}
\partial_{M^{2}} X_{1} & =\frac{-2 m e^{2}}{\pi^{2}} \int_{0}^{k_{F}} \frac{p^{2} d p\left(-p^{2}+k_{F}^{2}+M^{2}\right)}{\left(\left(p^{2}+k_{F}^{2}+M^{2}\right)^{2}-4 p^{2} k_{F}^{2}\right)^{2}}, \\
\partial_{M^{2}} U_{1} & =\frac{-e^{2}}{2 \pi^{2}} \int_{0}^{k_{F}} \frac{p^{2} d p}{\left(p^{2}+k_{F}^{2}+M^{2}\right)^{2}-4 p^{2} k_{F}^{2}} .
\end{aligned}
$$

The right hand side of these equations is $u_{0}$-independent, giving $X_{0, M^{2}}=\hbar, X_{1, M^{2}}\left(u_{0}\right)=X_{1, M^{2}}$ and $U_{1, M^{2}}\left(u_{0}\right)=$ $U_{1, M^{2}}=e u_{0}=0$ with

$$
\partial_{M^{2}} X_{1, M^{2}}=-\frac{m e^{2}}{8 \pi^{2}}\left\{\frac{1}{2 k_{F}^{3}} \ln \frac{M^{2}}{4 k_{F}^{2}+M^{2}}+\frac{1}{k_{F}\left(4 k_{F}^{2}+M^{2}\right)}+\frac{1}{k_{F} M^{2}}\right\}
$$

In order to obtain the expression for $X_{1, M^{2}}$, one has to integrate this diffential equation over $M^{2}$ from $M^{2} \rightarrow \infty$ to $\epsilon \rightarrow 0$. This yields

$$
X_{1, M^{2}}=-\frac{m e^{2}}{8 \pi^{2}} \frac{1}{k_{F}}\left(2+\lim _{\epsilon \rightarrow 0} \ln \frac{\epsilon}{4 k_{F}^{2}}\right)+\mathcal{O}\left(M^{-2}\right) .
$$

This coupling constant represents the ratio of the bare to the effective mass of the electron. It diverges as $\epsilon \rightarrow 0$ meaning that the electron effective mass vanishes. This is a well known result from perturbation theory [15] and corresponds to the Hartree-Fock approximation. Note that the divergence in the perturbation expansion is also logarithmic and appears as $\lim _{k \rightarrow k_{F}} \ln \left(k-k_{F}\right) / 2 k_{F}$. The singularity appearing when $k \rightarrow k_{F}$ corresponds in the $M$-parameter space to $M \rightarrow 0$.

\section{B. Fluctuations: Thomas-Fermi mass}

The reason to go beyond the homogeneous photon field approximation is the need of the kinetic energy for the photon to reproduce the desired non-analycity in the photon self energy at $\omega=p=0$. The terms $\mathcal{O}\left(\eta^{2}\right)$ in the evolution equation determine the inverse photon propagator,

$$
\begin{aligned}
\frac{1}{2} & \left(\partial_{M^{2}} Z_{0} \omega_{q}^{2}-\partial_{M^{2}} Z_{1} q^{2}-\partial_{M^{2}} U_{2}^{(2)}\right) \stackrel{\underline{\underline{g}}}{\underline{y}} \\
& -i \hbar f^{\prime} e^{2} \int \frac{d^{3} p}{(2 \pi)^{3}} \int \frac{d \omega_{p}}{2 \pi} \frac{\mathcal{E}_{p}}{\left(\hbar \omega_{p}-(1+f) \mathcal{E}_{p}+e u_{0}\right)^{2}\left(\hbar \omega_{p+q}-(1+f) \mathcal{E}_{p+q}+e u_{0}\right)} .
\end{aligned}
$$


The frequency integral yields

$$
\frac{i}{\hbar} \mathcal{E}_{p} \frac{-\Theta\left(-\mathcal{E}_{p}\right)+\Theta\left(-\mathcal{E}_{p+q}\right)}{\left(\hbar \omega_{q}-(1+f)\left(\mathcal{E}_{p+q}-\mathcal{E}_{p}\right)+i\left(\delta_{p+q}-\delta_{p}\right)\right)^{2}}
$$

which can be written as

$$
\frac{i}{\hbar} \mathcal{E}_{p}\left\{\frac{-\Theta\left(-\mathcal{E}_{p}\right) \Theta\left(\mathcal{E}_{p+q}\right)}{\left(\hbar \omega_{q}-(1+f)\left(\mathcal{E}_{p+q}-\mathcal{E}_{p}\right)+i \delta\right)^{2}}+\frac{\Theta\left(\mathcal{E}_{p}\right) \Theta\left(-\mathcal{E}_{p+q}\right)}{\left(\hbar \omega_{q}-(1+f)\left(\mathcal{E}_{p+q}-\mathcal{E}_{p}\right)-i \delta\right)^{2}}\right\}
$$

remembering that $\delta_{p}=\operatorname{sign}\left(\mathcal{E}_{p}\right) \delta$. Performing the change of variable $-p-q \rightarrow p$ in the second term, one finds

$$
\begin{aligned}
& \frac{1}{2}\left(\partial_{M^{2}} Z_{0} \omega_{q}^{2}-\partial_{M^{2}} Z_{1} q^{2}-\partial_{M^{2}} U_{2}^{(2)}\right) \underline{\underline{\underline{\underline{x}}}} f^{\prime} e^{2} \int \frac{d^{3} p}{(2 \pi)^{3}} \Theta\left(-\mathcal{E}_{p}\right)\left[1-\Theta\left(-\mathcal{E}_{p+q}\right)\right] \\
& \quad \times\left\{\frac{-\mathcal{E}_{p}}{\left(\hbar \omega_{q}-(1+f)\left(\mathcal{E}_{p+q}-\mathcal{E}_{p}\right)+i \delta\right)^{2}}+\frac{\mathcal{E}_{p+q}}{\left(\hbar \omega_{q}+(1+f)\left(\mathcal{E}_{p+q}-\mathcal{E}_{p}\right)-i \delta\right)^{2}}\right\} .
\end{aligned}
$$

The dependence on $\omega_{q}$ drops out as $q \rightarrow 0$, giving $Z_{0}=\hbar$.

In the following, the real and imaginary parts are treated separately. The imaginary part is easier to compute once the integration over $M^{2}$ has been performed. We find

$$
\pi e^{2} \int \frac{d^{3} p}{(2 \pi)^{3}} \frac{\Theta\left(-\mathcal{E}_{p}\right) \Theta\left(\mathcal{E}_{p+q}\right)}{\mathcal{E}_{p+q}-\mathcal{E}_{p}}\left\{\mathcal{E}_{p} \delta\left(\omega_{q}-\mathcal{E}_{p+q}+\mathcal{E}_{p}\right)-\mathcal{E}_{p+q} \delta\left(\omega_{q}+\mathcal{E}_{p+q}-\mathcal{E}_{p}\right)\right\}
$$

which is vanishing when $\omega_{q}=0$ is imposed.

The real part of the r.h.s. of Eq. (57) is simplified by noticing that the exchange $p \leftrightarrows p+q$ leads to the vanishing of the products of Heaviside step functions. Moreover, setting $\omega_{q}=0$ yields

$$
\partial_{M^{2}} Z_{1} q^{2}+\partial_{M^{2}} U_{2}^{(2)} \stackrel{\underline{\underline{g x}}}{\underline{2}}-2 e^{2} \frac{f^{\prime}}{(1+f)^{2}} \int \frac{d^{3} p}{(2 \pi)^{3}} \frac{\Theta\left(-\mathcal{E}_{p}\right)}{\left(\mathcal{E}_{p+q}-\mathcal{E}_{p}\right)} \stackrel{\underline{\underline{\mathrm{gr}}}}{-}-\frac{f^{\prime}}{(1+f)^{2}} \frac{m e^{2} k_{F}}{2 \pi^{2}} g\left(\frac{q}{k_{F}}\right)
$$

where

$$
g(x)=\frac{1}{2}-\frac{1}{2 x}\left(1-\frac{x^{2}}{4}\right) \ln \left|\frac{1-\frac{x}{2}}{1+\frac{x}{2}}\right|
$$

is the Lindhard function [15]. The integration over $M^{2}$ from $M \rightarrow \infty$ to 0 is easily computed. The expansion in powers of $q$ finally gives

$$
Z_{1, M^{2}}\left(u_{0}\right)=-\frac{m e^{2}}{24 \pi^{2} k_{F}}+\mathcal{O}\left(M^{-2}\right), \quad U_{2, M^{2}}\left(u_{0}\right)=\frac{m k_{F} e^{2}}{4 \pi^{2}} u_{0}^{2}+\mathcal{O}\left(M^{-2}\right) .
$$

The non-vanishing coefficient of $u_{0}^{2}, m k_{F} e^{2} / 4 \pi^{2}$, is the Thomas-Fermi mass giving the screening length in the electron gas. The other coefficient functions remain unchanged at the one-loop approximation.

\section{SUMMARY}

A new non-perturbative evolution scheme is developed in this paper for the electron gas with Coulomb interaction. No expansion in the electric charge is needed, the only approximation is the restriction of the evolution of the effective action to a given subspace of functionals, i.e. the consideration of a given set of 1PI functions with a given energymomentum dependence. The evolution equations, a system of coupled equations for the 1PI functions are to be integrated out numerically from the initial condition given by the tree-level action functional.

Since the evolution is performed by adjusting the amplitude of the quantum fluctuations there is no conflict between the successive elimination strategy of the renormalization group and the existence of a non-trivial Fermi surface.

The unique feature of this scheme is that by truncating the effective action at higher order in the fields or in the derivatives one obtains systematically improved, non-perturbative approximations. Since the computation is carried 
out in continuous space-time and the time can be chosen to be real this method offers an alternative for the usual numerical simulations of field theories on the lattice.

The method provides the solution of the theory in the sense that physically important quantities are easy to express in terms of 1PI functions, the final result of the evolution equation. The only approximation, the truncation of the effective action in the power of the fields and their gradient has transparent interpretation and can be controled numerically. In fact, the maximal power of a given field in the effective action gives order of the 1PI functions retained in "turning on" the interactions. The order of the gradient expansion corresponds to the order of the Taylor expansion of the 1PI functions around the Fermi surface and $p=\omega=0$ for fermions and bosons, respectively.

We considered in this work the independent mode solution of the evolution equation only. This approximation reproduces the one-loop results of the usual perturbation expansion without relying Feynman graphs. In order to go beyond well known results one has to consider the system of coupled differential equations for the coefficient functions in the effective action and integrate them out numerically. This work, the numerical determination of the transport coefficients for the Coulomb gas is in progress.

\section{ACKNOWLEDGMENTS}

This work is supported in part by the grant OTKA T29927/98.

[1] M. Gell-Mann, F. E. Low, Phys. Rev. 95, 1300 (1954).

[2] F. J. Wegner, A. Houghton, Phys. Rev. A 8, 401 (1975).

[3] C. Wetterich, Phys. Lett. B 301, 90 (1993).

[4] T. Morris, Int. J. Mod. Phys. B 9, 2411 (1994).

[5] J. Alexandre, J. Polonyi, Ann. Phys. 288, 37 (2001).

[6] C. G. Callan, Phys. Rev. D 2, 1541 (1970); K. Symanzik, Commun. Math. Phys. 18, 227 (1970).

[7] G. Benfatto, G. Gallavotti, J. Stat. Phys. 59, 541 (1991).

[8] J. Feldman, R. Trubowitz, Helv. Phys. Act. 63, 156 (1991); Helv. Phys. Act. 64, 213 (1991).

[9] J. Feldman, J. Magneu, V. Rivasseau, R. Trubowitz, Helv. Phys. Act. 65, 679 (1992); Helv. Phys. Act. 66, 498 (1993).

[10] R. Shankar, Rev. Mod. Phys. 66, 129 (1994).

[11] M. Salmhofer, Rev. Math. Phys. 10, 553 (1998).

[12] M. Kreutz, T. N. Tudron, Phys. Rev. D 16, 2978 (1977).

[13] S. F. Edwards, Philos. Mag. 3, 1020 (1958).

[14] D. Vollhardt, P. Wölfle, Phys. Rev. B 22, 4666 (1980).

[15] A. L. Fetter and J. D. Walecka, Quantum theory of many-paticles systems, McGraw-Hill, 1971.

\section{APPENDIX A: FUNCTIONAL DERIVATIVES}

For the right hand side of the evolution equation we find the second functional derivative matrix of the effective action, $\Gamma^{(2)}\left[\psi^{\dagger}, \psi, u\right]$. Since we expand the effective action in the fluctuations in order to identify the terms $\mathcal{O}\left(\partial^{2}\right)$ we ultimately need the functional derivatives up to fourth order.

The first four derivatives of the effective action (21) written as $\Gamma=\int_{x}\left(\psi_{x}^{\dagger} K_{x} \psi_{x}+L_{x}\right)$, are

$$
\begin{aligned}
\Gamma_{\psi_{y}}^{(1)}= & -\int_{x} \psi_{x}^{\dagger} K_{x} \delta_{x, y}, \\
\Gamma_{\psi_{y}^{\dagger}}^{(1)}= & \int_{x} \delta_{x, y} K_{x} \psi_{x}, \\
\Gamma_{u_{y}}^{(1)}= & \int_{x} \delta_{x, y}\left(\psi_{x}^{\dagger} K_{x}^{(1)} \psi_{x}+L_{x}^{(1)}\right)+\int_{x}\left\{\psi_{x}^{\dagger}\left[Y_{0} \partial_{t} u_{x} \partial_{t} \delta_{x, y}+Y_{1} \nabla u_{x} \nabla \delta_{x, y}\right] \psi_{x}\right. \\
& \left.+Z_{0} \partial_{t} u_{x} \partial_{t} \delta_{x, y}-Z_{1} \nabla u_{x} \nabla \delta_{x, y}\right\},
\end{aligned}
$$


where

$$
\begin{aligned}
K_{x}^{(n)} & =i X_{0}^{(n)}\left(u_{x}\right) \partial_{t}-X_{1}^{(n)}\left(u_{x}\right) \mathcal{E}+U_{1}^{(n)}\left(u_{x}\right)+\frac{1}{2} Y_{0}^{(n)}\left(u_{x}\right)\left(\partial_{t} u_{x}\right)^{2}+\frac{1}{2} Y_{1}^{(n)}\left(u_{x}\right)\left(\nabla u_{x}\right)^{2}, \\
L_{x}^{(n)} & =\frac{1}{2} Z_{0}^{(n)}\left(u_{x}\right)\left(\partial_{t} u_{x}\right)^{2}-\frac{1}{2} Z_{1}^{(n)}\left(u_{x}\right)\left(\nabla u_{x}\right)^{2}-U_{2}^{(n)}\left(u_{x}\right)
\end{aligned}
$$

and the coefficient functions $X, Y$ and $Z$ are considered at the value $u_{x}$ in the integrands.

The second derivatives,

$$
\begin{aligned}
\Gamma_{\psi_{y_{1}}^{\dagger} \psi_{y_{2}}}^{(2)}= & -\int_{x} \delta_{x, y_{1}} K_{x} \delta_{x, y_{2}}, \\
\Gamma_{\psi_{y_{1}} u_{y_{2}}}^{(2)}= & -\int_{x} \delta_{x, y_{2}} \psi_{x}^{\dagger} K_{x}^{(1)} \delta_{x, y_{1}}-\int_{x} \psi_{x}^{\dagger}\left[Y_{0} \partial_{t} u_{x} \partial_{t} \delta_{x, y_{2}}+Y_{1} \nabla u_{x} \nabla \delta_{x, y_{2}}\right] \delta_{x, y_{1}} \\
\Gamma_{\psi_{y_{1}}^{\dagger} u_{y_{2}}}^{(2)}= & \int_{x} \delta_{x, y_{1}} \delta_{x, y_{2}} K_{x}^{(1)} \psi_{x}+\int_{x} \delta_{x, y_{1}}\left[Y_{0} \partial_{t} u_{x} \partial_{t} \delta_{x, y_{2}}+Y_{1} \nabla u_{x} \nabla \delta_{x, y_{2}}\right] \psi_{x} \\
\Gamma_{u_{y_{1}} u_{y_{2}}}^{(2)}= & \int_{x} \delta_{x, y_{1}} \delta_{x, y_{2}}\left(\psi_{x}^{\dagger} K_{x}^{(2)} \psi_{x}+L_{x}^{(2)}\right) \\
& +\int_{x}\left(\psi_{x}^{\dagger} \psi_{x} Y_{0}^{(1)}+Z_{0}^{(1)}\right) \partial_{t} u_{x}\left(\delta_{x, y_{1}} \partial_{t} \delta_{x, y_{2}}+\delta_{x, y_{2}} \partial_{t} \delta_{x, y_{1}}\right) \\
& +\int_{x}\left(\psi_{x}^{\dagger} \psi_{x} Y_{1}^{(1)}-Z_{1}^{(1)}\right) \nabla u_{x}\left(\delta_{x, y_{1}} \nabla \delta_{x, y_{2}}+\delta_{x, y_{2}} \nabla_{t} \delta_{x, y_{1}}\right) \\
& +\int_{x}\left(\psi_{x}^{\dagger} \psi_{x} Y_{0}+Z_{0}\right) \partial_{t} \delta_{x, y_{1}} \partial_{t} \delta_{x, y_{2}}+\int_{x}\left(\psi_{x}^{\dagger} \psi_{x} Y_{1}-Z_{1}\right) \nabla \delta_{x, y_{1}} \nabla \delta_{x, y_{2}},
\end{aligned}
$$

yield the non-diagonal, fluctuation-independent contribution to the self energy,

$$
\Gamma_{\text {nd }}=\left(\begin{array}{ccc}
0 & 0 & \Sigma^{\psi^{\dagger} u} \\
0 & 0 & \Sigma^{\psi u} \\
\Sigma^{u \psi} & \Sigma^{u \psi^{\dagger}} & \Sigma^{u u}
\end{array}\right)
$$

with

$$
\begin{aligned}
& \Sigma_{p_{1}, p_{2}}^{u, \psi}=\Sigma_{p_{2}, p_{1}}^{\psi, u}=-\psi_{-p_{2}-p_{1}}^{\dagger} A_{p_{2}}^{(1)}, \\
& \Sigma_{p_{1}, p_{2}}^{u, \psi^{\dagger}}=\Sigma_{p_{2}, p_{1}}^{\psi^{\dagger}, u}=A_{-p_{1}-p_{2}}^{(1)} \psi_{-p_{1}-p_{2}}, \\
& \Sigma_{p_{1}, p_{2}}^{u, u}=\int_{q} B_{p_{1}, p_{2}}^{q} \psi_{-q-p_{1}-p_{2}}^{\dagger} \psi_{q},
\end{aligned}
$$

and

$$
B_{p_{1}, p_{2}}^{q}=A_{q}^{(2)}-Y_{0} \omega_{p_{1}} \omega_{p_{2}}-Y_{1} p_{1} \cdot p_{2},
$$

and $A$ being defined in Eq. (28). The coefficient functions $X, Y$ and $Z$ are considered at $u_{0}$ in the gradient expansion formulae.

In order to find the contributions of the fluctuations to the self energy up to the quadratic order we need the following functional derivatives,

$$
\begin{aligned}
& \Gamma_{\psi_{y_{1}}^{\dagger} \psi_{y_{2}} u_{y_{3}}}^{(3)}=-\int_{x} \delta_{x, y_{1}} \delta_{x, y_{3}} K_{x}^{(1)} \delta_{x, y_{2}}-\int_{x} \delta_{x, y_{1}}\left[Y_{0} \partial_{t} u_{x} \partial_{t} \delta_{x, y_{3}}+Y_{1} \nabla u_{x} \nabla \delta_{x, y_{3}}\right] \delta_{x, y_{2}},
\end{aligned}
$$

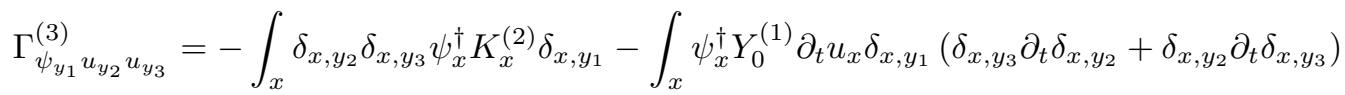

$$
\begin{aligned}
& -\int_{x} \psi_{x}^{\dagger} Y_{1}^{(1)} \nabla u_{x} \delta_{x, y_{1}}\left(\delta_{x, y_{3}} \nabla \delta_{x, y_{2}}+\delta_{x, y_{2}} \nabla \delta_{x, y_{3}}\right) \\
& -\int_{x} \psi_{x}^{\dagger}\left[Y_{0} \partial_{t} \delta_{x, y_{3}} \partial_{t} \delta_{x, y_{2}}+Y_{1} \nabla \delta_{x, y_{3}} \nabla \delta_{x, y_{2}}\right] \delta_{x, y_{1}},
\end{aligned}
$$




$$
\begin{aligned}
& \Gamma_{\psi_{y_{1}}^{\dagger} u_{y_{2}} u_{y_{3}}}^{(3)}=\int_{x} \delta_{x, y_{1}} \delta_{x, y_{2}} \delta_{x, y_{3}} K_{x}^{(2)} \psi_{x}+\int_{x} \delta_{x, y_{1}} Y_{0}^{(1)} \partial_{t} u_{x}\left(\delta_{x, y_{3}} \partial_{t} \delta_{x, y_{2}}+\delta_{x, y_{2}} \partial_{t} \delta_{x, y_{3}}\right) \psi_{x} \\
& +\int_{x} \delta_{x, y_{1}} Y_{1}^{(1)} \nabla u_{x}\left(\delta_{x, y_{3}} \nabla \delta_{x, y_{2}}+\delta_{x, y_{2}} \nabla \delta_{x, y_{3}}\right) \psi_{x} \\
& +\int_{x} \delta_{x, y_{1}}\left[Y_{0} \partial_{t} \delta_{x, y_{3}} \partial_{t} \delta_{x, y_{2}}+Y_{1} \nabla \delta_{x, y_{3}} \nabla \delta_{x, y_{2}}\right] \psi_{x} \\
& \Gamma_{u_{y_{1}} u_{y_{2}} u_{y_{3}}}^{(3)}=\int_{x} \delta_{x, y_{1}} \delta_{x, y_{2}} \delta_{x, y_{3}}\left(\psi_{x}^{\dagger} K_{x}^{(3)} \psi_{x}+L_{x}^{(3)}\right)+\int_{x}\left(\psi_{x}^{\dagger} \psi_{x} Y_{0}^{(2)}+Z_{0}^{(2)}\right) \partial_{t} u_{x} \\
& \times\left(\delta_{x, y_{2}} \delta_{x, y_{3}} \partial_{t} \delta_{x, y_{1}}+\delta_{x, y_{1}} \delta_{x, y_{3}} \partial_{t} \delta_{x, y_{2}}+\delta_{x, y_{1}} \delta_{x, y_{2}} \partial_{t} \delta_{x, y_{3}}\right) \\
& +\int_{x}\left(\psi_{x}^{\dagger} \psi_{x} Y_{1}^{(2)}-Z_{1}^{(2)}\right) \nabla u_{x} \\
& \times\left(\delta_{x, y_{2}} \delta_{x, y_{3}} \nabla \delta_{x, y_{1}}+\delta_{x, y_{1}} \delta_{x, y_{3}} \nabla \delta_{x, y_{2}}+\delta_{x, y_{1}} \delta_{x, y_{2}} \nabla \delta_{x, y_{3}}\right) \\
& +\int_{x}\left(\psi_{x}^{\dagger} \psi_{x} Y_{0}^{(1)}+Z_{0}^{(1)}\right) \\
& \times\left(\delta_{x, y_{1}} \partial_{t} \delta_{x, y_{2}} \partial_{t} \delta_{x, y_{3}}+\delta_{x, y_{2}} \partial_{t} \delta_{x, y_{3}} \partial_{t} \delta_{x, y_{1}}+\delta_{x, y_{3}} \partial_{t} \delta_{x, y_{1}} \partial_{t} \delta_{x, y_{2}}\right) \\
& +\int_{x}\left(\psi_{x}^{\dagger} \psi_{x} Y_{1}^{(1)}-Z_{1}^{(1)}\right) \\
& \times\left(\delta_{x, y_{1}} \nabla \delta_{x, y_{2}} \nabla \delta_{x, y_{3}}+\delta_{x, y_{2}} \nabla \delta_{x, y_{3}} \nabla \delta_{x, y_{1}}+\delta_{x, y_{3}} \nabla \delta_{x, y_{1}} \nabla \delta_{x, y_{2}}\right) \text {, } \\
& \Gamma_{\psi_{y_{1}}^{\dagger} \psi_{y_{2}} u_{y_{3}} u_{y_{4}}}^{(4)}=-\int_{x} \delta_{x, y_{1}} \delta_{x, y_{3}} \delta_{x, y_{4}} K_{x}^{(2)} \delta_{x, y_{2}}-\int_{x} \delta_{x, y_{1}} \delta_{x, y_{2}} Y_{0}^{(1)} \partial_{t} u_{x}\left(\delta_{x, y_{3}} \partial_{t} \delta_{x, y_{4}}+\delta_{x, y_{4}} \partial_{t} \delta_{x, y_{3}}\right) \\
& -\int_{x} \delta_{x, y_{1}} \delta_{x, y_{2}} Y_{1}^{(1)} \nabla u_{x}\left(\delta_{x, y_{3}} \nabla \delta_{x, y_{4}}+\delta_{x, y_{4}} \nabla \delta_{x, y_{3}}\right) \\
& -\int_{x} \delta_{x, y_{1}} \delta_{x, y_{2}}\left(Y_{0} \partial_{t} \delta_{x, y_{3}} \partial_{t} \delta_{x, y_{4}}+Y_{1} \nabla \delta_{x, y_{3}} \nabla \delta_{x, y_{4}}\right), \\
& \Gamma_{u_{y_{1}} u_{y_{2}} u_{y_{3}} \psi_{y_{4}}^{\dagger}}^{(4)}=\int_{x} \delta_{x, y_{1}} \delta_{x, y_{2}} \delta_{x, y_{3}} \delta_{x, y_{4}} K_{x}^{(3)} \psi_{x} \\
& +\int_{x} \psi_{x} \delta_{x, y_{4}} Y_{0}^{(2)} \partial_{t} u_{x}\left(\delta_{x, y_{2}} \delta_{x, y_{3}} \partial_{t} \delta_{x, y_{1}}+\delta_{x, y_{1}} \delta_{x, y_{3}} \partial_{t} \delta_{x, y_{2}}+\delta_{x, y_{1}} \delta_{x, y_{2}} \partial_{t} \delta_{x, y_{3}}\right) \\
& +\int_{x} \psi_{x} \delta_{x, y_{4}} Y_{1}^{(2)} \nabla u_{x}\left(\delta_{x, y_{2}} \delta_{x, y_{3}} \nabla \delta_{x, y_{1}}+\delta_{x, y_{1}} \delta_{x, y_{3}} \nabla \delta_{x, y_{2}}+\delta_{x, y_{1}} \delta_{x, y_{2}} \nabla \delta_{x, y_{3}}\right) \\
& +\int_{x} \psi_{x} \delta_{x, y_{4}} Y_{0}^{(1)}\left(\delta_{x, y_{1}} \partial_{t} \delta_{x, y_{2}} \partial_{t} \delta_{x, y_{3}}+\delta_{x, y_{2}} \partial_{t} \delta_{x, y_{3}} \partial_{t} \delta_{x, y_{1}}+\delta_{x, y_{3}} \partial_{t} \delta_{x, y_{1}} \partial_{t} \delta_{x, y_{2}}\right) \\
& +\int_{x} \psi_{x} \delta_{x, y_{4}} Y_{1}^{(1)}\left(\delta_{x, y_{1}} \nabla \delta_{x, y_{2}} \nabla \delta_{x, y_{3}}+\delta_{x, y_{2}} \nabla \delta_{x, y_{3}} \nabla \delta_{x, y_{1}}+\delta_{x, y_{3}} \nabla \delta_{x, y_{1}} \nabla \delta_{x, y_{2}}\right), \\
& \Gamma_{u_{y_{1}} u_{y_{2}} u_{y_{3}} \psi_{y_{4}}}^{(4)}=-\int_{x} \delta_{x, y_{1}} \delta_{x, y_{2}} \delta_{x, y_{3}} \psi_{x}^{\dagger} K_{x}^{(3)} \delta_{x, y_{4}} \\
& -\int_{x} \psi_{x}^{\dagger} \delta_{x, y_{4}} Y_{0}^{(2)} \partial_{t} u_{x}\left(\delta_{x, y_{2}} \delta_{x, y_{3}} \partial_{t} \delta_{x, y_{1}}+\delta_{x, y_{1}} \delta_{x, y_{3}} \partial_{t} \delta_{x, y_{2}}+\delta_{x, y_{1}} \delta_{x, y_{2}} \partial_{t} \delta_{x, y_{3}}\right) \\
& -\int_{x} \psi_{x}^{\dagger} \delta_{x, y_{4}} Y_{1}^{(2)} \nabla u_{x}\left(\delta_{x, y_{2}} \delta_{x, y_{3}} \nabla \delta_{x, y_{1}}+\delta_{x, y_{1}} \delta_{x, y_{3}} \nabla \delta_{x, y_{2}}+\delta_{x, y_{1}} \delta_{x, y_{2}} \nabla \delta_{x, y_{3}}\right) \\
& -\int_{x} \psi_{x}^{\dagger} \delta_{x, y_{4}} Y_{0}^{(1)}\left(\delta_{x, y_{1}} \partial_{t} \delta_{x, y_{2}} \partial_{t} \delta_{x, y_{3}}+\delta_{x, y_{2}} \partial_{t} \delta_{x, y_{3}} \partial_{t} \delta_{x, y_{1}}+\delta_{x, y_{3}} \partial_{t} \delta_{x, y_{1}} \partial_{t} \delta_{x, y_{2}}\right) \\
& -\int_{x} \psi_{x}^{\dagger} \delta_{x, y_{4}} Y_{1}^{(1)}\left(\delta_{x, y_{1}} \nabla \delta_{x, y_{2}} \nabla \delta_{x, y_{3}}+\delta_{x, y_{2}} \nabla \delta_{x, y_{3}} \nabla \delta_{x, y_{1}}+\delta_{x, y_{3}} \nabla \delta_{x, y_{1}} \nabla \delta_{x, y_{2}}\right), \\
& \Gamma_{u_{y_{1}} u_{y_{2}} u_{y_{3}} u_{y_{4}}}^{(4)}=\int_{x} \delta_{x, y_{1}} \delta_{x, y_{2}} \delta_{x, y_{3}} \delta_{x, y_{4}}\left(\psi_{x}^{\dagger} K_{x}^{(4)} \psi_{x}+L_{x}^{(4)}\right) \\
& +\int_{x}\left(\psi_{x}^{\dagger} \psi_{x} Y_{0}^{(3)}+Z_{0}^{(3)}\right) \partial_{t} u_{x}\left(\delta_{x, y_{2}} \delta_{x, y_{3}} \delta_{x, y_{4}} \partial_{t} \delta_{x, y_{1}}\right.
\end{aligned}
$$




$$
\begin{aligned}
& \left.+\delta_{x, y_{1}} \delta_{x, y_{3}} \delta_{x, y_{4}} \partial_{t} \delta_{x, y_{2}}+\delta_{x, y_{1}} \delta_{x, y_{2}} \delta_{x, y_{4}} \partial_{t} \delta_{x, y_{3}}+\delta_{x, y_{1}} \delta_{x, y_{2}} \delta_{x, y_{3}} \partial_{t} \delta_{x, y_{4}}\right) \\
& +\int_{x}\left(\psi_{x}^{\dagger} \psi_{x} Y_{1}^{(3)}-Z_{1}^{(3)}\right) \nabla u_{x}\left(\delta_{x, y_{2}} \delta_{x, y_{3}} \delta_{x, y_{4}} \nabla \delta_{x, y_{1}}\right. \\
& \left.+\delta_{x, y_{1}} \delta_{x, y_{3}} \delta_{x, y_{4}} \nabla \delta_{x, y_{2}}+\delta_{x, y_{1}} \delta_{x, y_{2}} \delta_{x, y_{4}} \nabla \delta_{x, y_{3}}+\delta_{x, y_{1}} \delta_{x, y_{2}} \delta_{x, y_{3}} \nabla \delta_{x, y_{4}}\right) \\
& +\int_{x}\left(\psi_{x}^{\dagger} \psi_{x} Y_{0}^{(2)}+Z_{0}^{(2)}\right)\left(\delta_{x, y_{1}} \delta_{x, y_{4}} \partial_{t} \delta_{x, y_{2}} \partial_{t} \delta_{x, y_{3}}\right. \\
& +\delta_{x, y_{2}} \delta_{x, y_{4}} \partial_{t} \delta_{x, y_{3}} \partial_{t} \delta_{x, y_{1}}+\delta_{x, y_{2}} \delta_{x, y_{3}} \partial_{t} \delta_{x, y_{1}} \partial_{t} \delta_{x, y_{4}}+\delta_{x, y_{1}} \delta_{x, y_{3}} \partial_{t} \delta_{x, y_{2}} \partial_{t} \delta_{x, y_{4}} \\
& \left.+\delta_{x, y_{1}} \delta_{x, y_{2}} \partial_{t} \delta_{x, y_{3}} \partial_{t} \delta_{x, y_{4}}+\delta_{x, y_{3}} \delta_{x, y_{4}} \partial_{t} \delta_{x, y_{1}} \partial_{t} \delta_{x, y_{2}}\right) \\
& +\int_{x}\left(\psi_{x}^{\dagger} \psi_{x} Y_{1}^{(2)}-Z_{1}^{(2)}\right)\left(\delta_{x, y_{1}} \delta_{x, y_{4}} \nabla \delta_{x, y_{2}} \nabla \delta_{x, y_{3}}+\delta_{x, y_{2}} \delta_{x, y_{4}} \nabla \delta_{x, y_{3}} \nabla \delta_{x, y_{1}}\right. \\
& +\delta_{x, y_{2}} \delta_{x, y_{3}} \nabla \delta_{x, y_{1}} \nabla \delta_{x, y_{4}}+\delta_{x, y_{1}} \delta_{x, y_{3}} \nabla \delta_{x, y_{2}} \nabla \delta_{x, y_{4}} \\
& \left.+\delta_{x, y_{1}} \delta_{x, y_{2}} \nabla \delta_{x, y_{3}} \nabla \delta_{x, y_{4}}+\delta_{x, y_{3}} \delta_{x, y_{4}} \nabla \delta_{x, y_{1}} \nabla \delta_{x, y_{2}}\right) .
\end{aligned}
$$

The matrix elements (34) needed for the right hand side of the evolution equation can be read off easily,

$$
\begin{aligned}
\sigma_{p_{1}, p_{2}}^{\psi^{\dagger} \psi}= & -\sigma_{p_{2}, p_{1}}^{\psi \psi_{1}^{\dagger}}=-A_{p_{2}}^{(1)} \eta_{-p_{1}-p_{2}}-\int_{q} C_{p_{1}, p_{2}}^{q} \eta_{-q-p_{1}-p_{2}} \eta_{q} \\
\sigma_{p_{1}, p_{2}}^{\psi^{\dagger} \psi^{\dagger}}= & \sigma_{p_{1}, p_{2}}^{\psi \psi}=0 \\
\sigma_{p_{1}, p_{2}}^{\psi^{\dagger} u}= & \sigma_{p_{2}, p_{1}}^{u \psi^{\dagger}}=\int_{q} D_{p_{1}, p_{2}}^{q} \eta_{q} \psi_{-q-p_{1}-p_{2}}+\int_{q_{1}, q_{2}} E_{p_{1}, p_{2}}^{q_{1}, q_{2}} \eta_{q_{1}} \eta_{q_{2}} \psi_{-q_{1}-q_{2}-p_{1}-p_{2}}, \\
\sigma_{p_{1}, p_{2}}^{\psi u}= & \sigma_{p_{2}, p_{1}}^{u \psi}=-\int_{q} F_{p_{1}, p_{2}}^{q} \eta_{q} \psi_{-q-p_{1}-p_{2}}^{\dagger}-\int_{q_{1}, q_{2}} G_{p_{1}, p_{2}}^{q_{1}, q_{2}} \eta_{q_{1}} \eta_{q_{2}} \psi_{-q_{1}-q_{2}-p_{1}-p_{2}}^{\dagger} \\
\sigma_{p_{1}, p_{2}}^{u u}= & -H_{p_{1}, p_{2}} \eta_{-p_{1}-p_{2}}+\int_{q_{1}, q_{2}} I_{p_{1}, p_{2}}^{q_{1}, q_{2}} \eta_{q_{1}} \psi_{q_{2}}^{\dagger} \psi_{-q_{1}-q_{2}-p_{1}-p_{2}} \\
& +\int_{q_{1}, q_{2}, q_{3}} J_{p_{1}, p_{2}}^{q_{1}, q_{2}, q_{3}} \eta_{q_{1}} \eta_{q_{2}} \psi_{q_{3}}^{\dagger} \psi_{-q_{1}-q_{2}-q_{3}-p_{1}-p_{2}}-\int_{q} K_{p_{1}, p_{2}}^{q} \eta_{q} \eta_{-q-p_{1}-p_{2}},
\end{aligned}
$$

where

$$
\begin{aligned}
C_{p_{1}, p_{2}}^{q}= & \frac{1}{2}\left[A_{p_{2}}^{(2)}-Y_{0} \omega_{q}\left(\omega_{q}+\omega_{p_{1}}+\omega_{p_{2}}\right)+Y_{1} q \cdot\left(q+p_{1}+p_{2}\right)\right] \\
D_{p_{1}, p_{2}}^{q}= & A_{-q-p_{1}-p_{2}}^{(2)}-Y_{0} \omega_{q} \omega_{p_{2}}-Y_{1} q \cdot p_{2}, \\
E_{p_{1}, p_{2}}^{q_{1}, q_{2}}= & \frac{1}{2}\left\{A_{-q_{1}-q_{2}-p_{1}-p_{2}}^{(3)}-Y_{0}^{(1)}\left[\omega_{q_{1}} \omega_{q_{2}}+\left(\omega_{q_{1}}+\omega_{q_{2}}\right) \omega_{p_{2}}\right]-Y_{1}^{(1)}\left[q_{1} \cdot q_{2}+\left(q_{1}+q_{2}\right) \cdot p_{2}\right]\right\}, \\
F_{p_{1}, p_{2}}^{q}= & A_{p_{1}}^{(2)}-Y_{0} \omega_{q} \omega_{p_{2}}-Y_{1} q \cdot p_{2}, \\
G_{p_{1}, p_{2}}^{q_{1}, q_{2}}= & \frac{1}{2}\left\{A_{p_{1}}^{(3)}-Y_{0}^{(1)}\left[\omega_{p_{2}}\left(\omega_{q_{1}}+\omega_{q_{2}}\right)+\omega_{q_{1}} \omega_{q_{2}}\right]-Y_{1}^{(1)}\left[p_{2} \cdot\left(q_{1}+q_{2}\right)+q_{1} \cdot q_{2}\right]\right\}, \\
H_{p_{1}, p_{2}}= & Z_{0}^{(1)}\left[\left(\omega_{p_{1}}+\omega_{p_{2}}\right)^{2}+\omega_{p_{1}} \omega_{p_{2}}\right]+Z_{1}^{(1)}\left[\left(p_{1}+p_{2}\right)^{2}+p_{1} \cdot p_{2}\right]+U_{2}^{(3)}, \\
I_{p_{1}, p_{2}}^{q_{1}, q_{2}}= & A_{-q_{1}-q_{2}-p_{1}-p_{2}}^{(3)}-Y_{0}^{(1)}\left[\omega_{q_{1}}\left(\omega_{p_{1}}+\omega_{p_{2}}\right)+\omega_{p_{1}} \omega_{p_{2}}\right]-Y_{1}^{(1)}\left[q_{1} \cdot\left(p_{1}+p_{2}\right)+p_{1} \cdot p_{2}\right], \\
J_{p_{1}, p_{2}}^{q_{1}, q_{2}, q_{3}}= & \frac{1}{2}\left\{A_{-q_{1}-q_{2}-q_{3}-p_{1}-p_{2}}^{(4)}-Y_{0}^{(2)}\left[\omega_{q_{1}} \omega_{q_{2}}+\left(\omega_{q_{1}}+\omega_{q_{2}}\right)\left(\omega_{p_{1}}+\omega_{p_{2}}\right)+\omega_{p_{1}} \omega_{p_{2}}\right]\right. \\
& \left.-Y_{1}^{(2)}\left[q_{1} \cdot q_{2}+\left(q_{1}+q_{2}\right) \cdot\left(p_{1}+p_{2}\right)+p_{1} \cdot p_{2}\right]\right\} \\
K_{p_{1}, p_{2}}^{q}= & \frac{1}{2}\left\{Z_{0}^{(2)}\left[\left(\omega_{q}+\omega_{p_{1}}+\omega_{p_{2}}\right)^{2}+\omega_{q}\left(\omega_{p_{1}}+\omega_{p_{2}}\right)+\omega_{p_{1}} \omega_{p_{2}}\right]\right. \\
& \left.+Z_{1}^{(2)}\left[\left(q+p_{1}+p_{2}\right)^{2}-q \cdot\left(p_{1}+p_{2}\right)-p_{1} \cdot p_{2}\right]+U_{2}^{(4)}\right\} .
\end{aligned}
$$




\section{APPENDIX B: GRADIENT EXPANSION}

Here we provide more detailed expressions for the gradient expansion of the evolution equation Eq. (17).

The fluctuations of the photon field to the evolution equation will be retaind up to the level $\mathcal{O}\left(\psi^{\dagger} \psi \eta^{2}\right)$. These contributions from the expansion (35) are inserted on the right hand side of the evolution equation Eq. (17). We introduce the notation

$$
\mathcal{F}_{n}=-\sum_{\alpha, \cdots, \alpha_{n}} \operatorname{Tr}\left[\mathcal{E} W_{\alpha_{1}, \cdots, \alpha_{n}, j, j^{\dagger}}\right], \quad \mathcal{B}_{n}=\sum_{\alpha, \cdots, \alpha_{n}} \operatorname{Tr}\left[W_{\alpha_{1}, \cdots, \alpha_{n}, J, J}\right],
$$

where the summation is over index sets where the number of value " $f$ " is 1 or 2 . We find for the necessary terms

$$
\begin{aligned}
& -\operatorname{Tr}\left[\mathcal{E} W_{\mathrm{fl}, j, j^{\dagger}}\right]=\int_{p} \mathcal{E}_{p} G_{p}^{2} \sigma_{-p, p}^{\psi \psi^{\dagger}} \\
& \mathcal{F}_{2}=-\int_{p, p^{\prime}} \mathcal{E}_{p} G_{p}^{2}\left(\Sigma_{-p, p^{\prime}}^{\psi u} D_{p^{\prime}} \sigma_{-p^{\prime}, p}^{u \psi^{\dagger}}+\sigma_{-p, p^{\prime}}^{\psi u} D_{p^{\prime}} \Sigma_{-p^{\prime}, p}^{u \psi^{\dagger}}+\sigma_{-p, p^{\prime}}^{\psi \psi^{\dagger}} G_{-p^{\prime}} \sigma_{-p^{\prime}, p}^{\psi \psi^{\dagger}}+\sigma_{-p, p^{\prime}}^{\psi u} D_{p^{\prime}} \sigma_{-p^{\prime}, p}^{u \psi^{\dagger}}\right), \\
& \mathcal{F}_{3}=\int_{p, p^{\prime}, p^{\prime \prime}} \mathcal{E}_{p} G_{p}^{2}\left(\sigma_{-p, p^{\prime}}^{\psi \psi^{\dagger}} G_{-p^{\prime}} \Sigma_{-p^{\prime}, p^{\prime \prime}}^{\psi u} D_{p^{\prime \prime}} \Sigma_{-p^{\prime \prime}, p}^{u \psi^{\dagger}}+\Sigma_{-p, p^{\prime}}^{\psi u} D_{p^{\prime}} \sigma_{-p^{\prime}, p^{\prime \prime}}^{u u} D_{p^{\prime \prime}} \Sigma_{-p^{\prime \prime}, p}^{u \psi^{\dagger}}\right. \\
& +\Sigma_{-p, p^{\prime}}^{\psi u} D_{p^{\prime}} \Sigma_{-p^{\prime}, p^{\prime \prime}}^{u \psi^{\dagger}} G_{-p^{\prime \prime}} \sigma_{-p^{\prime \prime}, p}^{\psi \psi^{\dagger}}+\sigma_{-p, p^{\prime}}^{\psi \psi^{\dagger}} G_{-p^{\prime}} \sigma_{-p^{\prime}, p^{\prime \prime}}^{\psi u} D_{p^{\prime \prime}} \Sigma_{-p^{\prime \prime}, p}^{u \psi^{\dagger}}+\sigma_{-p, p^{\prime}}^{\psi u} D_{p^{\prime}} \sigma_{-p^{\prime}, p^{\prime \prime}}^{u u} D_{p^{\prime \prime}} \Sigma_{-p^{\prime \prime}, p}^{u \psi^{\dagger}} \\
& \left.+\sigma_{-p, p^{\prime}}^{\psi \psi^{\dagger}} G_{-p^{\prime}} \Sigma_{-p^{\prime}, p^{\prime \prime}}^{\psi u} D_{p^{\prime \prime}} \sigma_{-p^{\prime \prime}, p}^{u \psi^{\dagger}}+\Sigma_{-p, p^{\prime}}^{\psi u} D_{p^{\prime}} \sigma_{-p^{\prime}, p^{\prime \prime}}^{u \psi^{\dagger}} G_{-p^{\prime \prime}} \sigma_{-p^{\prime \prime}, p}^{\psi \psi^{\dagger}}+\Sigma_{-p, p^{\prime}}^{\psi u} D_{p^{\prime}} \sigma_{-p^{\prime}, p^{\prime \prime}}^{u u} D_{p^{\prime \prime}} \sigma_{-p^{\prime \prime}, p}^{u \psi^{\dagger}}\right) \text {, } \\
& \mathcal{F}_{4}=\int_{p, p^{\prime}, p^{\prime \prime}, p^{\prime \prime \prime}} \mathcal{E}_{p} G_{p}^{2}\left(-\sigma_{-p, p^{\prime}}^{\psi \psi^{\dagger}} G_{-p^{\prime}} \sigma_{-p^{\prime}, p^{\prime \prime}}^{\psi \psi^{\dagger}} G_{-p^{\prime \prime}} \Sigma_{-p^{\prime \prime}, p^{\prime \prime \prime}}^{\psi u} D_{p^{\prime \prime \prime}} \Sigma_{-p^{\prime \prime \prime}, p}^{u \psi^{\dagger}}\right. \\
& -\sigma_{-p, p^{\prime}}^{\psi \psi^{\dagger}} G_{-p^{\prime}} \Sigma_{-p^{\prime}, p^{\prime \prime}}^{\psi u} D_{p^{\prime \prime}} \sigma_{-p^{\prime \prime}, p^{\prime \prime \prime}}^{u u} D_{p^{\prime \prime \prime}} \Sigma_{-p^{\prime \prime \prime}, p}^{u \psi^{\dagger}}-\Sigma_{-p, p^{\prime}}^{\psi u} D_{p^{\prime}} \sigma_{-p^{\prime}, p^{\prime \prime}}^{u u} D_{p^{\prime \prime}} \sigma_{-p^{\prime \prime}, p^{\prime \prime \prime}}^{u u} D_{p^{\prime \prime \prime}} \Sigma_{-p^{\prime \prime \prime}, p}^{u \psi^{\dagger}} \\
& \left.+\Sigma_{-p, p^{\prime}}^{\psi u} D_{p^{\prime}} \sigma_{-p^{\prime}, p^{\prime \prime}}^{u u} D_{p^{\prime \prime}} \Sigma_{-p^{\prime \prime}, p^{\prime \prime \prime}}^{u \psi^{\dagger}} G_{p^{\prime \prime \prime}} \sigma_{-p^{\prime \prime \prime}, p}^{\psi \psi^{\dagger}}-\Sigma_{-p, p^{\prime}}^{\psi u} D_{p^{\prime}} \Sigma_{-p^{\prime}, p^{\prime \prime}}^{u \psi^{\dagger}} G_{-p^{\prime \prime}} \sigma_{-p^{\prime \prime}, p^{\prime \prime \prime}}^{\psi \psi^{\dagger}} G_{-p^{\prime \prime \prime}} \sigma_{-p^{\prime \prime \prime}, p}^{\psi \psi^{\dagger}}\right),
\end{aligned}
$$

for the electron contributions and

$$
\begin{aligned}
& \operatorname{Tr}\left[W_{\mathrm{fl}, J, J}\right]=-\int_{p} D_{p}^{2} \sigma_{-p, p}^{u u} \\
& \mathcal{B}_{2}=\int_{p, p^{\prime}} D_{p}^{2}\left(-2 \Sigma_{-p, p^{\prime}}^{u \psi} G_{p^{\prime}} \sigma_{-p^{\prime}, p}^{\psi^{\dagger} u}+2 \Sigma_{-p, p^{\prime}}^{u \psi^{\dagger}} G_{-p^{\prime}} \sigma_{-p^{\prime}, p}^{\psi u}+2 \Sigma_{-p, p^{\prime}}^{u u} D_{p^{\prime}} \sigma_{-p^{\prime}, p}^{u u}\right. \\
& \left.-\sigma_{-p, p^{\prime}}^{u \psi} G_{p^{\prime}} \sigma_{-p^{\prime}, p}^{\psi^{\dagger} u}+\sigma_{-p, p^{\prime}}^{u \psi^{\dagger}} G_{-p^{\prime}} \sigma_{-p^{\prime}, p}^{\psi u}+\sigma_{-p, p^{\prime}}^{u u} D_{p^{\prime}} \sigma_{-p^{\prime}, p}^{u u}\right), \\
& \mathcal{B}_{3}=\int_{p, p^{\prime}, p^{\prime \prime}} D_{p}^{2}\left(\sigma_{-p, p^{\prime}}^{u u} D_{p^{\prime}} \Sigma_{-p^{\prime}, p^{\prime \prime}}^{u \psi} G_{p^{\prime \prime}} \Sigma_{-p^{\prime \prime}, p}^{\psi^{\dagger} u}-\sigma_{-p, p^{\prime}}^{u u} D_{p^{\prime}} \Sigma_{-p^{\prime}, p^{\prime \prime}}^{u \psi^{\dagger}} G_{-p^{\prime \prime}} \Sigma_{-p^{\prime \prime}, p}^{\psi u}+\Sigma_{-p, p^{\prime}}^{u \psi} G_{p^{\prime}} \Sigma_{-p^{\prime}, p}^{\psi^{\dagger} u} D_{p^{\prime \prime}} \sigma_{-p^{\prime \prime}, p}^{u u}\right. \\
& -\Sigma_{-p, p^{\prime}}^{u \psi^{\dagger}} G_{-p^{\prime}} \Sigma_{-p^{\prime}, p}^{\psi u} D_{p^{\prime \prime}} \sigma_{-p^{\prime \prime}, p}^{u u}+\Sigma_{-p, p^{\prime}}^{u \psi} G_{p^{\prime}} \sigma_{-p^{\prime}, p^{\prime \prime}}^{\psi^{\dagger} u} D_{p^{\prime \prime}} \sigma_{-p^{\prime \prime}, p}^{u u}-\Sigma_{-p, p^{\prime}}^{u \psi^{\dagger}} G_{-p^{\prime}} \sigma_{-p^{\prime}, p^{\prime \prime}}^{\psi u} D_{p^{\prime \prime}} \sigma_{-p^{\prime \prime}, p}^{u u} \\
& +\sigma_{-p, p^{\prime}}^{u u} D_{p^{\prime}} \Sigma_{-p^{\prime}, p^{\prime \prime}}^{u \psi} G_{p^{\prime \prime}} \sigma_{-p^{\prime \prime}, p}^{\psi^{\dagger} u}-\sigma_{-p, p^{\prime}}^{u u} D_{p^{\prime}} \Sigma_{-p^{\prime}, p^{\prime \prime}}^{u \psi^{\dagger}} G_{-p^{\prime \prime}} \sigma_{-p^{\prime \prime}, p}^{\psi u}+\sigma_{-p, p^{\prime}}^{u \psi} G_{p^{\prime}} \Sigma_{-p^{\prime}, p^{\prime \prime}}^{\psi^{\dagger} u} D_{p^{\prime \prime}} \sigma_{-p^{\prime \prime}, p}^{u u} \\
& \left.-\sigma_{-p, p^{\prime}}^{u \psi^{\dagger}} G_{-p^{\prime}} \Sigma_{-p^{\prime}, p^{\prime \prime}}^{\psi u} D_{p^{\prime \prime}} \sigma_{-p^{\prime \prime}, p}^{u u}+\sigma_{-p, p^{\prime}}^{u u} D_{p^{\prime}} \sigma_{-p^{\prime}, p^{\prime \prime}}^{u \psi} G_{p^{\prime \prime}} \Sigma_{-p^{\prime \prime}, p}^{\psi^{\dagger} u}-\sigma_{-p, p^{\prime}}^{u u} D_{p^{\prime}} \sigma_{-p^{\prime}, p^{\prime \prime}}^{u \psi^{\dagger}} G_{-p^{\prime \prime}} \Sigma_{-p^{\prime \prime}, p}^{\psi u}\right), \\
& \mathcal{B}_{4}=\int_{p, p^{\prime}, p^{\prime \prime}, p^{\prime \prime \prime}} D_{p}^{2}\left(2 \sigma_{-p, p^{\prime}}^{u u} D_{p^{\prime}} \sigma_{-p^{\prime}, p^{\prime \prime}}^{u u} D_{p^{\prime \prime}} \Sigma_{-p^{\prime \prime}, p^{\prime \prime \prime}}^{u \psi^{\dagger}} G_{-p^{\prime \prime \prime}} \Sigma_{-p^{\prime \prime \prime}, p}^{\psi u}\right. \\
& -2 \sigma_{-p, p^{\prime}}^{u u} D_{p^{\prime}} \sigma_{-p^{\prime}, p^{\prime \prime}}^{u u} D_{p^{\prime \prime}} \Sigma_{-p^{\prime \prime}, p^{\prime \prime \prime}}^{u \psi} G_{p^{\prime \prime \prime}} \Sigma_{-p^{\prime \prime \prime}, p}^{\psi^{\dagger} u} \\
& +2 \sigma_{-p, p^{\prime}}^{u u} D_{p^{\prime}} \Sigma_{-p^{\prime}, p^{\prime \prime}}^{u \psi^{\dagger}} G_{-p^{\prime \prime}} \sigma_{-p^{\prime \prime}, p^{\prime \prime \prime}}^{\psi \psi^{\dagger}} G_{-p^{\prime \prime \prime}} \Sigma_{-p^{\prime \prime \prime}, p}^{\psi u}+2 \sigma_{-p, p^{\prime}}^{u u} D_{p^{\prime}} \Sigma_{-p^{\prime}, p^{\prime \prime}}^{u \psi} G_{p^{\prime \prime}} \sigma_{-p^{\prime \prime}, p^{\prime \prime \prime}}^{\psi^{\dagger} \psi} G_{p^{\prime \prime \prime}} \Sigma_{-p^{\prime \prime \prime}, p}^{\psi^{\dagger} u} \\
& +\sigma_{-p, p^{\prime}}^{u u} D_{p^{\prime}} \Sigma_{-p^{\prime}, p^{\prime \prime}}^{u \psi^{\dagger}} G_{-p^{\prime \prime}} \Sigma_{-p^{\prime \prime}, p^{\prime \prime \prime}}^{\psi u} D_{p^{\prime \prime \prime}} \sigma_{-p^{\prime \prime \prime}, p}^{u u}-\sigma_{-p, p^{\prime}}^{u u} D_{p^{\prime}} \Sigma_{-p^{\prime}, p^{\prime \prime}}^{u \psi} G_{p^{\prime \prime}} \Sigma_{-p^{\prime \prime}, p^{\prime \prime \prime}}^{\psi^{\dagger} u} D_{p^{\prime \prime \prime}} \sigma_{-p^{\prime \prime \prime}, p}^{u u} \\
& \left.+\Sigma_{-p, p^{\prime}}^{u \psi^{\dagger}} G_{-p^{\prime}} \sigma_{-p^{\prime}, p^{\prime \prime}}^{\psi \psi^{\dagger}} G_{-p^{\prime \prime}} \sigma_{-p^{\prime \prime}, p^{\prime \prime \prime}}^{\psi \psi^{\dagger}} G_{-p^{\prime \prime \prime}} \Sigma_{-p^{\prime \prime \prime}, p}^{\psi u}-\Sigma_{-p, p^{\prime}}^{u \psi} G_{p^{\prime}} \sigma_{-p^{\prime}, p^{\prime \prime}}^{\psi^{\dagger} \psi} G_{p^{\prime \prime}} \sigma_{-p^{\prime \prime}, p^{\prime \prime \prime}}^{\psi^{\dagger} \psi} G_{p^{\prime \prime \prime}} \Sigma_{-p^{\prime \prime}, p}^{\psi^{\dagger} u}\right),
\end{aligned}
$$

for the photon induced terms. As discussed below Eq. (43) the sum of the terms listed in Eqs. (B2) and (B3) yields the evolution for $Y_{0}$ and $Y_{1}$. 